\title{
Non-periodic finite-element formulation of Kohn-Sham Density Functional Theory
}

\author{
Phanish Suryanarayana ${ }^{a}$ Vikram Gavini ${ }^{b}$ Thomas Blesgen ${ }^{c}$ \\ Kaushik Bhattacharya ${ }^{a}, *$ Michael Ortiz ${ }^{a}$ \\ ${ }^{\mathrm{a}}$ Division of Engineering and Applied Science, California Institute of Technology, CA \\ 91125, USA \\ ${ }^{\mathrm{b}}$ Department of Mechanical Engineering, University of Michigan, MI 48109-2125, USA \\ ${ }^{\mathrm{c}}$ Max Planck Institute for Mathematics in the Sciences, Inselstraße 22-26, D-04103 \\ Leipzig, Germany
}

\begin{abstract}
We present a real-space, non-periodic, finite-element formulation for Kohn-Sham Density Functional Theory (KS-DFT). We transform the original variational problem into a local saddle-point problem, and show its well-posedness by proving the existence of minimizers. Further, we prove the convergence of finite-element approximations including numerical quadratures. Based on domain decomposition, we develop a parallel finite-element implementation of this formulation capable of performing both all-electron and pseudopotential calculations. We assess the accuracy of the formulation through selected test cases and demonstrate good agreement with the literature. We also evaluate the numerical performance of the implementation with regard to its scalability and convergence rates. We view this work as a step towards developing a method that can accurately study defects like vacancies, dislocations and crack tips using Density Functional Theory (DFT) at reasonable computational cost by retaining electronic resolution where it is necessary and seamlessly coarse-graining far away.
\end{abstract}

Key words: Finite-elements; Kohn-Sham; Density Functional Theory; $\Gamma$-convergence

\section{Introduction}

The Schrödinger equation is fundamental for describing the quantum mechanical electronic structure of matter, since it does not require any empirical input. However, the solution of the Schrödinger equation is exceedingly expensive, and this limits the size

\footnotetext{
* Corresponding Author (bhatta@ caltech.edu)
} 
of systems that can be directly evaluated to tens of electrons (Kohn (1999)). Numerous approaches have been proposed to reduce the computational cost of the solution of the Schrödinger equation. These approaches include the widely used DFT of Hohenberg and Kohn (1964). In their seminal work, Hohenberg and Kohn (1964) proved the existence of a oneto-one correspondence between the ground state electron density and the ground state wavefunction of a many-particle system. By this correspondence, the electron density replaces the many-body electronic wavefunction as the fundamental unknown field, thereby greatly reducing the dimensionality and computational complexity of the problem.

The most common present-day implementation of DFT is through the Kohn-Sham method (Kohn and Sham (1965)), in which the intractable many-body problem of interacting electrons is reduced to a tractable problem of non-interacting electrons moving in an effective potential. Hidden in this formulation is the unknown exchange and correlation functional for which various models including the local spin-density approximation (LSDA) (Kohn and Sham (1965)) and the generalized gradient approximation (GGA) (Langreth and Mehl (1983); Perdew et al. (1992)) are used.

The plane-wave basis is one of the most-frequently used basis for solving the KohnSham problem (Kresse and Furthmüller (1996); Segall et al. (2002); Gonze et al. (2002); Ismail-Beigi and Arias (2000)) because of a number of attractive features. It forms a complete and orthonormal set that is independent of the atomic positions and is efficient for evaluating convolutions through the Fast Fourier Transform (FFT). However, the planewave basis also suffers from a few notable disadvantages. Firstly, the plane-wave basis is best suited to periodic systems. Therefore, the study of non-periodic and localized systems such as defects, clusters and surfaces requires the introduction of artificial supercell periodicity, which can lead to spurious results. Secondly, the plane-wave basis functions are non-local in real space, resulting in dense matrices which are ill-suited to iterative solution schemes. In addition, non-locality limits the usefulness of a planewave basis in multiscale approaches formulated in real space. Over the past decade, numerous efforts have been directed towards the development of real-space DFT implementations that overcome these difficulties (e. g., Hehre et al. (1969); Wills and Cooper (1987); Soler et al. (2002); Skylaris et al. (2005); Bowler et al. (2006); Chelikowsky et al. (1994); Castro et al. (2006)). Though some of these studies use the finite-element basis (Pask et al. (1999); Tsuchida (2004)), they do not utilize the unstructured nature of the the finite-element method and the convergence of the finite-element approximation with numerical quadratures has not been rigorously justified. This is one of the motivations of the current work along with the desire to coarse-grain KS-DFT.

The computational complexity of KS-DFT poses a significant hurdle in the solution of large systems of interest-irrespective of the basis set. Various approaches have been proposed to overcome this limitation. A few examples include the integration of DFT with molecular dynamics (Car and Parrinello (1985)), linear and sub-linear scaling algorithms (García-Cervera et al. (2007); Garcia-Cervera et al. (2009); Goedecker (1999); Galli and Parrinello (1992); Mauri et al. (1993); Skylaris et al. (2005)) and adaptive-coordinate systems (Gygi and Galli (1995); Zumbach et al. (1996); Modine et al. (1997)) which im- 
prove the efficiency of the real space calculations by selectively having some regions of space with higher resolution than others. Multiscale approaches which coarse-grain DFT or embed it into simpler, less accurate models like tight-binding (TB) or empirical potentials have also been developed recently (Choly et al. (2005); Lu et al. (2006); Govind et al. (1999); Bernstein et al. (2009)). Though these multiscale methods provide valuable insight, they suffer from a few notable drawbacks. In some cases, there is no seamless transition from DFT to TB or empirical potentials, while in others, uncontrolled approximations made by the use of linear response theory or Cauchy Born hypothesis render them unreliable. Also, there is no systematic convergence of the solution of these models to the full DFT solution.

In this paper, we present a non-periodic, real-space, finite-element formulation and implementation of KS-DFT. The present work builds on, and provides an extension of the likewise non-periodic, real-space, finite-element formulation of Gavini et al. (2007b) for orbital-free DFT (OFDFT). For definiteness, we develop the formulation for the LSDA exchange-correlation functional. However, the formulation is not restricted to a particular type of exchange-correlation functional and can be adapted to other commonly used functionals such as GGA. We intend to use the present real-space KS-DFT formulation as a stepping stone towards our final goal of studying localized defects in crystals such as vacancies, dislocations and cracks following in part our previous work with OFDFT (Gavini et al. (2007a)).

The approaches adopted for solving the Kohn-Sham problem can be broadly classified as ones that use the Self-Consistent Field (SCF) method and others in which some form of constrained direct minimization is performed (Payne et al. (1992)). However, these two approaches are equivalent (Parr and Yang (1989)) and largely a matter of convenience, as shown in Section 3.1 for completeness. Our method of solution utilizes both SCF and variational schemes to ensure convergence and achieve the fastest possible convergence rate. One of the most time-consuming parts of conventional real-space implementations is the evaluation of the non-local electrostatic interactions, which we overcome by computing the electrostatic potential directly. This direct computation of the electrostatic field has the effect of turning the original minimization problem into a saddle-point problem (Ismail-Beigi and Arias (2000)). The Lagrangian functional thus defined is subsequently discretized by means of finite-elements and numerical quadrature. We show in Section 3.2 that the saddle-point problem is mathematically well-posed by proving existence of solutions, and we prove the $\Gamma$-convergence of finite-element approximations with numerical quadratures thereof, in Sections 3.3 and 3.4. We also present a similar analysis for the pseudopotential approximation in Section 3.5. Our approach in Sections 3.2 through 3.4 follows that of our previous work with OFDFT (Gavini et al. (2007b)). However, there are additional technical difficulties that need to be addressed in the Kohn-Sham setting which we deal with here.

For completeness, we discuss in some detail relevant aspects of the finite-element implementation that are specific to the present application, including matters of optimal-mesh design, solution procedures (Section 4) and the computation of forces (Appendix A). In 
Section 5, we verify the formulation and numerical implementation by means of selected examples ranging from single atoms to small clusters. The core electrons are eliminated through the use of the Evanescent-Core (Fiolhais et al. (1995)) and Troullier-Martins (Troullier and Martins (1991)) pseudopotentials. To test the accuracy of the method, we also perform all-electron calculations for selected atoms and molecules. We also assess matters of numerical performance of the implementation including convergence rates, scaling with problem size and parallel scalability.

\section{Formulation}

The theoretical framework of DFT has its origins in the Hohenberg-Kohn theorems (Hohenberg and Kohn (1964)). The first theorem is related to uniqueness and states that the ground state expectation value of any observable is a unique functional of the ground state electron density. The second theorem sheds light on the variational structure of the problem and shows that the electron density that minimizes the total energy is the exact ground state density. The problem of finding the ground state energy and electron density is equivalent to the problem of minimizing the energy of a system of non-interacting electrons in a mean-field. The corresponding energy functional $\mathcal{E}: X \times \mathbb{R}^{S M} \rightarrow \mathbb{R}$ where $X$ is a suitable space of solutions for the orthogonal wavefunctions is given by (Parr and Yang (1989); Finnis (2003))

$$
\mathcal{E}(\Psi, \mathbf{R})=T_{\mathrm{s}}\left(\rho_{\alpha}, \rho_{\beta}\right)+E_{\mathrm{xc}}\left(\rho_{\alpha}, \rho_{\beta}\right)+E_{\mathrm{H}}\left(\rho_{\alpha}+\rho_{\beta}\right)+E_{\mathrm{ext}}\left(\rho_{\alpha}+\rho_{\beta}, \mathbf{R}\right)+E_{\mathrm{zz}}(\mathbf{R}),
$$

where

$$
\begin{aligned}
& \rho_{\alpha}(\mathbf{x})=\sum_{i=1}^{N_{\alpha}} \psi_{i \alpha}^{*}(\mathbf{x}) \psi_{i \alpha}(\mathbf{x})=\sum_{i=1}^{N_{\alpha}}\left|\psi_{i \alpha}(\mathbf{x})\right|^{2}, \\
& \rho_{\beta}(\mathbf{x})=\sum_{i=1}^{N_{\beta}} \psi_{i \beta}^{*}(\mathbf{x}) \psi_{i \beta}(\mathbf{x})=\sum_{i=1}^{N_{\beta}}\left|\psi_{i \beta}(\mathbf{x})\right|^{2}
\end{aligned}
$$

represent the electron densities with the spin component 'up' and 'down' respectively and $\rho(\mathbf{x})=\rho_{\alpha}(\mathbf{x})+\rho_{\beta}(\mathbf{x})$ is the total electron density. By $\Psi=\left\{\psi_{1 \alpha}, \psi_{2 \alpha}, \ldots, \psi_{N_{\alpha} \alpha}, \psi_{1 \beta}, \psi_{2 \beta}\right.$, $\left.\ldots, \psi_{N_{\beta} \beta}\right\}$, we denote the vector of wavefunctions and $\mathbf{R} \in \mathbb{R}^{S M}$ is the collection of all the nuclear positions $\mathbf{R}=\left\{\mathbf{R}_{1}, \mathbf{R}_{2}, \ldots, \mathbf{R}_{M}\right\}, S=3$ denotes the space dimension and $M \in \mathbb{N}$ is the number of nuclei. The wavefunctions are orthonormal, thereby satisfying the relation

$$
\int \psi_{i \sigma}^{*}(\mathbf{x}) \psi_{j \sigma}(\mathbf{x}) \mathrm{d} \mathbf{x}=\delta_{i j}, \quad \sigma \in\{\alpha, \beta\}, \quad i, j=1,2, \ldots, N_{\sigma},
$$

and

$$
N_{\alpha}=\int \rho_{\alpha}(\mathbf{x}) \mathrm{d} \mathbf{x}, \quad N_{\beta}=\int \rho_{\beta}(\mathbf{x}) \mathrm{d} \mathbf{x}
$$


represent the number of electrons with spin 'up' and 'down' respectively. Note that if the domain of integration is not specified, it refers to all of space $\mathbb{R}^{S}$. The first term

$$
T_{\mathrm{s}}\left(\rho_{\alpha}, \rho_{\beta}\right)=-\frac{1}{2} \sum_{\sigma} \sum_{i=1}^{N_{\sigma}} \int \psi_{i \sigma}^{*}(\mathbf{x}) \nabla^{2} \psi_{i \sigma}(\mathbf{x}) \mathrm{d} \mathbf{x}
$$

in Eqn. (1) is the kinetic energy of the non-interacting electrons. The terms

$$
\begin{aligned}
E_{\mathrm{H}}(\rho) & =\frac{1}{2} \iint \frac{\rho(\mathbf{x}) \rho\left(\mathbf{x}^{\prime}\right)}{\left|\mathbf{x}-\mathbf{x}^{\prime}\right|} \mathrm{d} \mathbf{x} \mathrm{d} \mathbf{x}^{\prime}, \\
E_{\mathrm{ext}}(\rho, \mathbf{R}) & =\int \rho(\mathbf{x}) V_{\mathrm{ext}}(\mathbf{x}, \mathbf{R}) \mathrm{d} \mathbf{x} \\
E_{\mathrm{zz}}(\mathbf{R}) & =\frac{1}{2} \sum_{I=1}^{M} \sum_{\substack{J=1 \\
J \neq I}}^{M} \frac{Z_{I} Z_{J}}{\left|\mathbf{R}_{I}-\mathbf{R}_{J}\right|}
\end{aligned}
$$

are electrostatic terms with $E_{\mathrm{H}}$ known as the Hartree energy representing the classical electrostatic interaction energy of the electron density, $E_{\text {ext }}$ is the interaction energy with the external potential $V_{\text {ext }}$ induced by the nuclear charges and $E_{\mathrm{zz}}$ denotes the repulsive energy between the nuclei. The term $E_{\mathrm{xc}}\left(\rho_{\alpha}, \rho_{\beta}\right)$ in Eqn. (1) denotes the exchangecorrelation energy. For definiteness, we specifically adopt the so-called LSDA in which the exchange-correlation energy is taken to be that of a uniform electron gas having the same local density (Kohn and Sham (1965)). The exchange-correlation energy can in turn be separated into individual contributions from the exchange and correlation parts, namely,

$$
E_{\mathrm{xc}}\left(\rho_{\alpha}, \rho_{\beta}\right)=E_{\mathrm{x}}\left(\rho_{\alpha}, \rho_{\beta}\right)+E_{\mathrm{c}}\left(\rho_{\alpha}, \rho_{\beta}\right)
$$

The expression for the exact exchange energy $E_{\mathrm{x}}\left(\rho_{\alpha}, \rho_{\beta}\right)$ of an uniform electron gas is

$$
E_{\mathrm{x}}\left(\rho_{\alpha}, \rho_{\beta}\right)=-\frac{3}{4}\left(\frac{6}{\pi}\right)^{1 / 3} \int\left(\rho_{\alpha}^{4 / 3}(\mathbf{x})+\rho_{\beta}^{4 / 3}(\mathbf{x})\right) \mathrm{d} \mathbf{x} .
$$

In addition, the correlation energy $E_{\mathrm{c}}\left(\rho_{\alpha}, \rho_{\beta}\right)$ can be written as

$$
E_{\mathrm{c}}\left(\rho_{\alpha}, \rho_{\beta}\right)=\int \varepsilon_{\mathrm{c}}\left(\rho_{\alpha}(\mathbf{x}), \rho_{\beta}(\mathbf{x})\right) \rho(\mathbf{x}) \mathrm{d} \mathbf{x} .
$$

Specifically, in applications we use the parametrization of Perdew and Wang (1992) fitted to accurate Monte Carlo simulations carried out by Ceperley and Alder (1980).

As expressed in Eqns. (7) and (9), the electrostatic interaction energy and the repulsive energy of the nuclei are non-local in nature and, thus are not amenable to a local discretization. In order to overcome this difficulty, we employ the following strategy. We begin by representing the nuclear charge of magnitude $Z_{I}$ at a site $R_{I} \in \mathbb{R}^{S}$ by means of a regularized bounded charge distribution $-Z_{I} \delta_{R_{I}}(\mathrm{x})$ with a compact support in a neighborhood of a small ball around $R_{I}$ (it is conventional in electronic-structure calculations to associate a negative charge with nuclei and a positive charge with electrons) and such 
that $\int_{\mathbb{R}^{S}} \delta_{R_{I}}(\mathbf{x}) \mathrm{d} \mathbf{x}=1$ for $1 \leq I \leq M$. We note that the electrostatic potential due to these regularized charge distributions is pointwise bounded. Defining as

$$
b(\mathbf{x}, \mathbf{R})=-\sum_{I=1}^{M} Z_{I} \delta_{R_{I}}(\mathbf{x})
$$

the sum of all such regularized charge distributions of the $M$ nuclei present in the system, the nuclear repulsive energy can be rewritten as

$$
E_{\mathrm{zz}}(\mathbf{R})=\frac{1}{2} \iint \frac{b(\mathbf{x}, \mathbf{R}) b\left(\mathbf{x}^{\prime}, \mathbf{R}\right)}{\left|\mathbf{x}-\mathbf{x}^{\prime}\right|} \mathrm{d} \mathbf{x} \mathrm{d} \mathbf{x}^{\prime}
$$

Notice that this differs from Eqn. (9) by the self-energy of the nuclei, but this is an inconsequential constant depending only on the nuclear charges. In addition, the electrostatic potential due to the nuclei and electron charge distribution can be computed as a solution to the Poisson equation

$$
\frac{-1}{4 \pi} \nabla^{2} \phi(\mathbf{x}, \mathbf{R})=\rho(\mathbf{x})+b(\mathbf{x}, \mathbf{R})
$$

This equation has the unique solution

$$
\phi(\mathbf{x}, \mathbf{R})=\int \frac{\rho\left(\mathbf{x}^{\prime}\right)}{\left|\mathbf{x}-\mathbf{x}^{\prime}\right|} \mathrm{d} \mathbf{x}^{\prime}+\int \frac{b\left(\mathbf{x}^{\prime}, \mathbf{R}\right)}{\left|\mathbf{x}-\mathbf{x}^{\prime}\right|} \mathrm{d} \mathbf{x}^{\prime}=V_{\mathrm{H}}(\mathbf{x})+V_{\mathrm{ext}}(\mathbf{x}, \mathbf{R}) .
$$

Consequently, we have the variational problem

$$
\begin{aligned}
& \frac{1}{2} \iint \frac{\rho(\mathbf{x}) \rho\left(\mathbf{x}^{\prime}\right)}{\left|\mathbf{x}-\mathbf{x}^{\prime}\right|} \mathrm{d} \mathbf{x} \mathrm{d} \mathbf{x}^{\prime}+\int \rho(\mathbf{x}) V_{\mathrm{ext}}(\mathbf{x}) \mathrm{d} \mathbf{x}+\frac{1}{2} \iint \frac{b(\mathbf{x}) b\left(\mathbf{x}^{\prime}\right)}{\left|\mathbf{x}-\mathbf{x}^{\prime}\right|} \mathrm{d} \mathbf{x} \mathrm{d} \mathbf{x}^{\prime} \\
& =-\inf _{\phi \in H_{0}^{1}\left(\mathbb{R}^{S}\right)}\left\{\frac{1}{8 \pi} \int|\nabla \phi(\mathbf{x}, \mathbf{R})|^{2} \mathrm{~d} \mathbf{x}-\int(\rho(\mathbf{x})+b(\mathbf{x}, \mathbf{R})) \phi(\mathbf{x}, \mathbf{R}) \mathrm{d} \mathbf{x}\right\} .
\end{aligned}
$$

Using this property, we can write Eqn. (1) as

$$
\mathcal{E}(\Psi, \mathbf{R})=\sup _{\phi \in H_{0}^{1}\left(\mathbb{R}^{S}\right)} \mathcal{L}(\Psi, \mathbf{R}, \phi)
$$

where

$$
\begin{aligned}
\mathcal{L}(\Psi, \mathbf{R}, \phi)= & -\frac{1}{2} \sum_{\sigma} \sum_{i=1}^{N_{\sigma}} \int \psi_{i \sigma}^{*}(\mathbf{x}) \nabla^{2} \psi_{i \sigma}(\mathbf{x}) \mathrm{d} \mathbf{x}+E_{\mathrm{xc}}\left(\rho_{\alpha}, \rho_{\beta}\right) \\
& -\frac{1}{8 \pi} \int|\nabla \phi(\mathbf{x}, \mathbf{R})|^{2} \mathrm{~d} \mathbf{x}+\int(\rho(\mathbf{x})+b(\mathbf{x}, \mathbf{R})) \phi(\mathbf{x}, \mathbf{R}) \mathrm{d} \mathbf{x} .
\end{aligned}
$$

Here and below, we denote by $H^{m}(\Omega)$ the space of $m$-times weakly differentiable functions in $L^{2}(\Omega)$ and by $H_{0}^{m}(\Omega):=\overline{C_{0}^{\infty}(\Omega)}{ }^{\|\cdot\|_{H}}$, the space of Sobolev functions with zero boundary conditions in the trace sense. 
The problem of determining the ground state electron density and the equilibrium positions of the nuclei can now be expressed as the saddle point problem

$$
\inf _{\substack{\Psi \in\left(H_{0}^{1}\left(\mathbb{R}^{S}\right)\right)^{N} \\ \mathbf{R} \in \mathbb{R}^{S M}}} \mathcal{E}(\Psi, \mathbf{R})=\inf _{\substack{\Psi \in\left(H_{0}^{1}\left(\mathbb{R}^{S}\right)\right)^{N} \\ \mathbf{R} \in \mathbb{R}^{S M}}} \sup _{\phi \in H_{0}^{1}\left(\mathbb{R}^{S}\right)} \mathcal{L}(\Psi, \mathbf{R}, \phi)
$$

subject to the constraints

$$
\int \psi_{i \sigma}^{*}(\mathbf{x}) \psi_{j \sigma}(\mathbf{x}) \mathrm{d} \mathbf{x}=\delta_{i j}, \quad \sigma \in\{\alpha, \beta\}, \quad i, j=1,2, \ldots, N_{\sigma} .
$$

In addition, $N_{\alpha}, N_{\beta}$ must be chosen such that the system has the lowest possible energy subject to the constraint of the total number of the electrons in the system being studied, i. e., $N_{\alpha}+N_{\beta}=N$.

\section{Properties of the Kohn-Sham variational problem}

In the present section, for the sake of clarity and notational simplicity, we will neglect spin polarization, thereby reducing the LSDA to the Local Density Approximation (LDA). This simplification is not essential, and the results presented in this section extend trivially to LSDA. Corresponding to Eqn. (1), for LDA we have

$$
\mathcal{E}(\Psi, \mathbf{R})=-\frac{1}{2} \sum_{i=1}^{N} \int \psi_{i}^{*}(\mathbf{x}) \nabla^{2} \psi_{i}(\mathbf{x}) \mathrm{d} \mathbf{x}+E_{\mathrm{xc}}(\rho)+E_{\mathrm{H}}(\rho)+E_{\mathrm{ext}}(\rho, \mathbf{R})+E_{\mathrm{zz}}(\mathbf{R})
$$

where $\rho(\mathbf{x})=\sum_{i=1}^{N}\left|\psi_{i}(\mathbf{x})\right|^{2}$ and $\Psi=\left\{\psi_{1}, \psi_{2}, \ldots, \psi_{N}\right\}$. Also, to avoid technical problems, we restrict our analysis to a bounded set $\Omega \subset \mathbb{R}^{S}$. With this simplification we obtain the variational problem

$$
\inf _{\substack{\Psi \in\left(H_{0}^{1}(\Omega)\right)^{N} \\ \mathbf{R} \in \mathbb{R}^{S M}}} \mathcal{E}(\Psi, \mathbf{R})=\inf _{\substack{\Psi \in\left(H_{0}^{1}(\Omega)\right)^{N} \\ \mathbf{R} \in \mathbb{R}^{S M}}} \sup _{\phi \in H_{0}^{1}(\Omega)} \mathcal{L}(\Psi, \mathbf{R}, \phi)
$$

subject to the constraints

$$
\int_{\Omega} \psi_{i}^{*}(\mathbf{x}) \psi_{j}(\mathbf{x}) \mathrm{d} \mathbf{x}=\delta_{i j}, \quad i, j=1,2, \ldots, N .
$$

Note that in this section we do not fix $S$, the dimension of space.

The remainder of this section has been organized as follows. In Section 3.1, we show the equivalence of the Kohn-Sham variational and eigenvalue problems. The existence of a minimum for the Kohn-Sham variational principle is proved in Section 3.2 and in Sections 3.3, 3.4 we validate the convergence of the finite-element approximation including numerical quadratures using the technique of $\Gamma$-convergence. $\Gamma$-convergence is an ideal and flexible tool for handling nonlinear variational problems like the one described by 
Eqns. (23), (24). Since a limitation of this method is its inability to provide the rates of convergence, we obtain it numerically in Section 5.4. The nonlinearity of the problem prevents the application of any of the standard techniques which provide the rate of convergence (Ciarlet (2002)).

\subsection{Equivalence of variational and eigenvalue problems}

We begin by showing the equivalence of the variational and eigenvalue problems in this section. The proof is similar in spirit to Roothaan (1951), where the equivalence was proved for the Hartree-Fock equations, and is provided here for the sake of completeness. Using Lagrange multipliers $\lambda_{i j}$ to enforce the constraints given by Eqn. (24), we obtain the functional

$$
\mathcal{E}_{c}(\Psi, \mathbf{R}, \Lambda)=\mathcal{E}(\Psi, \mathbf{R})-\sum_{i=1}^{N} \sum_{j=1}^{N} \lambda_{i j}\left(\int_{\Omega} \psi_{i}^{*}(\mathbf{x}) \psi_{j}(\mathbf{x}) \mathrm{d} \mathbf{x}-\delta_{i j}\right) .
$$

Let us first prove that the matrix $\Lambda=\left(\Lambda_{i j}\right)_{1 \leq i, j \leq N}$, whose entries are $\lambda_{i j}$, is Hermitian. Taking variations of Eqn. (25) and setting them to zero we obtain

$$
\begin{aligned}
\left(-\frac{1}{2} \nabla^{2}+V_{\mathrm{eff}}(\mathbf{x}, \mathbf{R})\right) \psi_{i}(\mathbf{x}) & =\sum_{j=1}^{N} \lambda_{i j} \psi_{j}(\mathbf{x}) \\
\left(-\frac{1}{2} \nabla^{2}+V_{\mathrm{eff}}(\mathbf{x}, \mathbf{R})\right) \psi_{i}^{*}(\mathbf{x}) & =\sum_{j=1}^{N} \lambda_{j i} \psi_{j}^{*}(\mathbf{x}) \\
\int_{\Omega} \psi_{i}^{*}(\mathbf{x}) \psi_{j}(\mathbf{x}) \mathrm{d} \mathbf{x} & =\delta_{i j}
\end{aligned}
$$

where

$$
V_{\text {eff }}(\mathbf{x}, \mathbf{R})=V_{\text {ext }}(\mathbf{x}, \mathbf{R})+V_{\mathrm{H}}(\mathbf{x})+\frac{\delta E_{\mathrm{xc}}(\rho)}{\delta \rho(\mathbf{x})} .
$$

Taking the complex conjugate of Eqn. (27), then subtracting it from Eqn. (26), we obtain the relation

$$
\sum_{j=1}^{N}\left(\lambda_{i j}-\lambda_{j i}^{*}\right) \psi_{j}(\mathbf{x})=0
$$

Since the $\psi_{j}$ are linearly independent it follows that $\lambda_{i j}=\lambda_{j i}^{*}$, showing the matrix $\Lambda$ to be indeed Hermitian. For notational convenience, we may express Eqn. (26) in matrix form as

$$
\mathcal{H} \Psi=\Psi \Lambda, \quad \mathcal{H}=-\frac{1}{2} \nabla^{2}+V_{\text {eff }}(\mathbf{x}, \mathbf{R}) .
$$

Now let us subject the wavefunctions to an unitary transformation and the matrix $\Lambda$ to a similarity transformation, i. e.,

$$
\hat{\Psi}=\Psi Q, \quad \hat{\Lambda}=Q^{*} \Lambda Q
$$


with $Q Q^{*}=Q^{*} Q=\mathbf{I}, \mathbf{I}$ being the identity matrix. Since unitary transformations are norm-conserving, the electron density remains invariant:

$$
\rho(\mathbf{x})=\sum_{i=1}^{N}\left|\psi_{i}(\mathbf{x})\right|^{2}=\sum_{i=1}^{N}\left|\hat{\psi}_{i}(\mathbf{x})\right|^{2} .
$$

Using the above relations we get

$$
\hat{\mathcal{H}} \hat{\Psi}=\hat{\Psi} \hat{\Lambda}
$$

where $\mathcal{H}=\hat{\mathcal{H}}$. Hence the wavefunctions on unitary transformation satisfy equations of exactly the same form as the original ones prior to transformation. Since the matrix $\Lambda$ is Hermitian, there exists an unitary matrix $Q$ such that $\hat{\Lambda}$ is a real diagonal matrix. Therefore, without any loss of generality we can replace Eqn. (31) by the eigenvalue problem

$$
\mathcal{H} \psi_{i}=\epsilon_{i} \psi_{i}, \quad i=1,2, \ldots N .
$$

which is said to be in canonical form. Thus, the SCF eigenvalue problem and the direct variational formulation of the problem are equivalent and the choice of one over other is strictly a matter of convenience.

\subsection{Existence of a minimum}

In this section, we establish the existence of a minimum for the Kohn-Sham variational principle. The main result is Theorem 4. A more general proof of the same is given by Anantharaman and Cances (2008), where they do not make the assumption that $\Omega$ is bounded. However, our analysis differs from theirs and is necessary as it lays the groundwork for proving the convergence of the finite-element approximation including numerical quadrature in the subsequent sections.

We introduce a suitable space of solutions $X$ corresponding to normalized orthogonal wavefunctions as

$$
X=\left\{\tilde{\Psi} \in\left(H_{0}^{1}(\Omega)\right)^{N} \mid\left\langle\tilde{\psi}_{i}, \tilde{\psi}_{j}\right\rangle_{\left(L^{2}(\Omega), L^{2}(\Omega)\right)}=\delta_{i j}, \quad i, j=1,2, \ldots, N\right\} .
$$

We require that $\Omega$ be an open, bounded subset of $\mathbb{R}^{S}$ with Lipschitz boundary. We point out that if we additionally postulate that $\Omega$ is a dyadic cube, then all the subsequent results of this analysis also hold for

$$
X=\left\{\tilde{\Psi} \in\left(H_{\mathrm{per}}^{1}(\Omega)\right)^{N} \mid\left\langle\tilde{\psi}_{i}, \tilde{\psi}_{j}\right\rangle_{\left(L^{2}(\Omega), L^{2}(\Omega)\right)}=\delta_{i j}, \quad i, j=1,2, \ldots, N\right\},
$$

which corresponds to a periodic system. By $H_{\text {per }}^{1}(\Omega)$ we denote the space of Sobolev functions on the torus obtained by identifying the opposite sides of $\Omega$.

On integrating by parts, the energy of the system as expressed by Eqn. (22) can be rewrit- 
ten in a generic form as

$$
\begin{array}{r}
\mathcal{E}(\Psi, \mathbf{R})=\sum_{i=1}^{N} \frac{1}{2} \int_{\Omega}\left|\nabla \psi_{i}(\mathbf{x})\right|^{2} \mathrm{~d} \mathbf{x}+J(\rho, \mathbf{R})+G(\rho), \quad \rho(\mathbf{x})=\sum_{i=1}^{N}\left|\psi_{i}(\mathbf{x})\right|^{2}, \\
J(\rho, \mathbf{R})=-\min _{\phi \in H_{0}^{1}(\Omega)}\left\{\frac{1}{C_{S}} \int_{\Omega}|\nabla \phi(\mathbf{x}, \mathbf{R})|^{2} \mathrm{~d} \mathbf{x}-\int_{\Omega}(\rho(\mathbf{x})+b(\mathbf{x}, \mathbf{R})) \phi(\mathbf{x}, \mathbf{R}) \mathrm{d} \mathbf{x}\right\}+\Sigma, \\
G(\rho)=\int_{\Omega} g(\rho) \mathrm{d} \mathbf{x},
\end{array}
$$

where $C_{S}$ is a constant dependent on the dimension of space $S$ and the term $\Sigma$ specifies the self energy of the nuclear charges which is an inconsequential constant in this analysis and hence will be dropped later.

We make the following hypothesis on $g$ :

(A1) The density $g$ is continuous in $\mathbb{R}^{+}$.

(A2) The growth condition $|g(t)| \leq c_{2}|t|^{q}+c_{1}$ holds for positive real constants $c_{2}, c_{1}$ and the exponent satisfies

$$
q \in \begin{cases}{\left[1, \frac{S}{S-2}\right),} & \text { if } S>2, \\ {[1,+\infty),} & \text { otherwise. }\end{cases}
$$

(A3) The exponent $q$ satisfies the condition $2 q<3$.

The need for Assumption (A3) becomes clear in the proof of Lemma 3.

Let the dual exponent $p^{*}$ of $p$ be given by $1 / p^{*}=1 / p-1 / S$. We first note:

(a) For $\rho \in L^{2}(\Omega)$ the functional $J(\rho, \mathbf{R})$ is well defined. This result follows from the Poincaré inequality and the Lax-Milgram Lemma. It is also straightforward to check that $\rho \in L^{2}(\Omega)$ if $2^{*}>4$.

(b) The functional $J$ is continuous in $L^{2}(\Omega)$.

(c) The functional $G$ is continuous in $L^{q}(\Omega)$, which follows from the continuity of $g$ and the growth condition (A2).

(d) As $\sum_{i=1}^{N} \frac{1}{2} \int_{\Omega}\left|\nabla \psi_{i}(\mathbf{x})\right|^{2} \mathrm{~d} \mathbf{x}$ is continuous in the strong topology of $\left(H_{0}^{1}(\Omega)\right)^{N}$ and convex, it follows that $\Psi \mapsto \sum_{i=1}^{N} \frac{1}{2} \int_{\Omega}\left|\nabla \psi_{i}(\mathbf{x})\right|^{2} \mathrm{dx}$ is lower semi-continuous in the weak topology of $\left(H_{0}^{1}(\Omega)\right)^{N}$. 
Lemma $1 X$ is closed in the weak topology of $\left(H_{0}^{1}(\Omega)\right)^{N}$.

Proof. Consider an arbitrary sequence $\left(\Psi_{l}\right)_{l} \subset X$ with $\Psi_{l} \rightarrow \Psi$ in $\left(H_{0}^{1}(\Omega)\right)^{N}$. By the Rellich-Kondrakov theorem (Rudin (1991)), $H_{0}^{1}(\Omega)$ has a compact embedding into $L^{2}(\Omega)$. Thus $\left(\psi_{i, l}\right)_{l} \rightarrow \psi_{i}$ in $L^{2}(\Omega)$ for $i=1,2, \ldots, N$ from which it follows that $\delta_{i j}=\left\langle\psi_{i, l} \mid \psi_{j, l}\right\rangle_{\left(L^{2}(\Omega), L^{2}(\Omega)\right)} \rightarrow\left\langle\psi_{i} \mid \psi_{j}\right\rangle_{\left(L^{2}(\Omega), L^{2}(\Omega)\right)}$ for $i, j=1,2, \ldots, N$ as $l \rightarrow \infty$. Therefore $\Psi \in X$, which implies that $X$ is closed in the weak topology of $\left(H_{0}^{1}(\Omega)\right)^{N}$.

Lemma 2 Let (A1), (A2) hold and $S<4$. Then $\mathcal{E}$ is lower semi-continuous in the weak topology of $X$.

Proof. Consider any sequence $\left(\Psi_{l}\right)_{l} \subset\left(H_{0}^{1}(\Omega)\right)^{N}$ such that $\Psi_{l} \rightarrow \Psi$ in $\left(H_{0}^{1}(\Omega)\right)^{N}$. As by the Sobolev theorem the embedding $H_{0}^{1}(\Omega) \hookrightarrow L^{4}(\Omega)$ is compact for $S<4$, it follows that $\left(\psi_{i, l}\right)_{l} \rightarrow \psi_{i}$ in $L^{4}(\Omega)$ for $i=1,2, \ldots, N$. Hence $\left(\psi_{i, l}^{2}\right)_{l} \rightarrow \psi_{i}^{2}$ in $L^{2}(\Omega)$ and therefore $\left(\rho_{l}\right)_{l} \rightarrow \rho$ in $L^{2}(\Omega)$. From (A2) it follows that the embedding $H_{0}^{1}(\Omega) \hookrightarrow L^{2 q}(\Omega)$ is compact. Thus $\rho_{l} \rightarrow \rho$ in $L^{\max \{2, q\}}(\Omega)$. From (b)-(d) it follows that $\sum_{i=1}^{N} \frac{1}{2} \int_{\Omega}\left|\nabla \psi_{i}(\mathbf{x})\right|^{2} \mathrm{~d} \mathbf{x}$, $J(\rho, \mathbf{R})$, and $G(\rho)$ are lower semi-continuous in the weak topology of $\left(H_{0}^{1}(\Omega)\right)^{N}$. Hence, it follows that $\mathcal{E}$ is lower semi-continuous in the weak topology of $\left(H_{0}^{1}(\Omega)\right)^{N}$, and as $X \subset\left(H_{0}^{1}(\Omega)\right)^{N}$, the claimed lower semi-continuity of $\mathcal{E}$ in the weak topology of $X$ follows.

Lemma 3 Let (A2), (A3) hold. Then $\mathcal{E}$ is coercive in the weak topology of $X$.

Proof. We first note the following results on the properties of $J(\rho, \mathbf{R})$ :

(i) From the linearity of the Euler-Lagrange equations associated with $J(\rho, \mathbf{R})$, the electrostatic energy of the system can be rewritten as

$$
\begin{aligned}
J(\rho, \mathbf{R}) & =E_{H}(\rho)+\int_{\Omega} V_{\text {ext }}(\mathbf{x}, \mathbf{R}) \rho(\mathbf{x}) \mathrm{d} \mathbf{x}+L(\mathbf{R}), \\
E_{H}(\rho) & =-\min _{\phi \in H_{0}^{1}(\Omega)}\left\{\frac{1}{C_{S}} \int_{\Omega}|\nabla \phi(\mathbf{x})|^{2} \mathrm{~d} \mathbf{x}-\int_{\Omega} \rho(\mathbf{x}) \phi(\mathbf{x}) \mathrm{d} \mathbf{x}\right\},
\end{aligned}
$$

where $L(\mathbf{R})=E_{z z}(\mathbf{R})+\Sigma$.

We point out that $V_{\text {ext }}$ is pointwise bounded. More precisely, it holds $\left|V_{\text {ext }}(\cdot, \mathbf{R})\right| \leq C$ almost everywhere in $\Omega$ for all $\mathbf{R} \in \mathbb{R}^{S M}$ and some constant $C$.

(ii) The functional $E_{H}$ is super-linear, that is $E_{H}\left(\rho_{1}+\rho_{2}\right) \geq E_{H}\left(\rho_{1}\right)+E_{H}\left(\rho_{2}\right)$ for arbitrary, almost everywhere positive $\rho_{1}, \rho_{2} \in L^{2}(\Omega)$. This follows from the linearity of the corresponding Euler-Lagrange equations.

From (ii) we can directly derive a lower bound for $E_{H}(\rho)$, 


$$
\begin{aligned}
E_{H}(\rho) & =E_{H}\left(\sum_{i=1}^{N}\left|\psi_{i}\right|^{2}\right) \geq \sum_{i=1}^{N} E_{H}\left(\left|\psi_{i}\right|^{2}\right) \\
& \geq \sum_{i=1}^{N}\left[\max _{\phi_{i} \in H_{0}^{1}(\Omega)}\left\{\int_{\Omega}\left|\psi_{i}(\mathbf{x})\right|^{2} \phi_{i}(\mathbf{x}) \mathrm{d} \mathbf{x}-\frac{1}{C_{S}} \int_{\Omega}\left|\nabla \phi_{i}(\mathbf{x})\right|^{2} \mathrm{~d} \mathbf{x}\right\}\right] .
\end{aligned}
$$

We use $\phi_{i}=C_{0}\left|\psi_{i}\right|$ as test functions in Eqn. (40) for a constant $C_{0}$ that will be determined later. Additionally, we also recall the following simple result on the weak derivative of an absolute function, whose proof is elementary but can for instance be found in Gilbarg and Trudinger (1983). If $\psi_{i} \in H^{1}(\Omega)$, then $\left|\psi_{i}\right| \in H^{1}(\Omega)$ and $\left\|\nabla\left|\psi_{i}\right|\right\|_{L^{2}(\Omega)} \leq$ $\left\|\nabla \psi_{i}\right\|_{L^{2}(\Omega)}$. Inserting $\phi_{i}(\mathbf{x})=C_{0}\left|\psi_{i}(\mathbf{x})\right|$ in Eqn. (40) as trial functions for $i=1,2, \ldots, N$, we arrive at the following lower bound for $E_{H}(\rho)$,

$$
\begin{aligned}
E_{H}(\rho) & \geq \sum_{i=1}^{N}\left[\max _{\phi_{i} \in H_{0}^{1}(\Omega)}\left\{\int_{\Omega}\left|\psi_{i}(\mathbf{x})\right|^{2} \phi_{i}(\mathbf{x}) \mathrm{d} \mathbf{x}-\frac{1}{C_{S}} \int_{\Omega}\left|\nabla \phi_{i}(\mathbf{x})\right|^{2} \mathrm{~d} \mathbf{x}\right\}\right] \\
& \geq \sum_{i=1}^{N}\left[C_{0} \int_{\Omega}\left|\psi_{i}(\mathbf{x})\right|^{3} \mathrm{~d} \mathbf{x}-\frac{C_{0}}{C_{S}} \int_{\Omega}\left|\nabla \psi_{i}(\mathbf{x})\right|^{2} \mathrm{~d} \mathbf{x}\right] \\
& =C_{0}\|\Psi\|_{L^{3}(\Omega)}^{3}-\frac{C_{0}}{C_{S}}\|\nabla \Psi\|_{L^{2}(\Omega)}^{2} .
\end{aligned}
$$

Using the inequality given by Eqn. (41) in Eqn. (39a) and $\left\|V_{\text {ext }}\right\|_{L^{\infty}(\Omega)} \leq C$ we conclude

$$
J(\rho) \geq C_{0}\|\Psi\|_{L^{3}(\Omega)}^{3}-\frac{C_{0}}{C_{S}}\|\nabla \Psi\|_{L^{2}(\Omega)}^{2}-C\|\Psi\|_{L^{2}(\Omega)}^{2} .
$$

Finally we require the inequality

$$
G(\rho) \geq-c_{0}\|\Psi\|_{L^{2 q(\Omega)}}^{2 q}-c_{1}
$$

This is a consequence of the growth condition (A2) on $g$ which yields

$$
G(\rho) \geq-c_{2}\left\|\sum_{i=1}^{N}\left|\psi_{i}\right|^{2}\right\|_{L^{q}(\Omega)}^{q}-c_{1}
$$

and a direct estimate of the norm in Eqn. (44).

With the help of Eqn. (42) and Eqn. (43) we end up with

$$
\mathcal{E}(\Psi, \mathbf{R}) \geq \frac{1}{2}\left(1-\frac{2 C_{0}}{C_{S}}\right)\|\nabla \Psi\|_{L^{2}(\Omega)}^{2}+C_{0}\|\Psi\|_{L^{3}(\Omega)}^{3}-c_{0}\|\Psi\|_{L^{2 q}(\Omega)}^{2 q}-C\|\Psi\|_{L^{2}(\Omega)}^{2}-c_{1} .
$$

Choosing $0<2 C_{0}<C_{S}$, we find that $\|\Psi\|_{L^{3}(\Omega)}^{3}$ grows faster than $\|\Psi\|_{L^{2 q}(\Omega)}^{2 q}$ as $2 q<3$ by (A3), and $\|\Psi\|_{L^{2}(\Omega)}^{2}$. This ensures $\mathcal{E}(\Psi) \rightarrow+\infty$ as $\|\Psi\|_{\left(H_{0}^{1}(\Omega)\right)^{N}} \rightarrow+\infty$. This is the 
coercivity of $\mathcal{E}$ in the weak topology of $\left(H_{0}^{1}(\Omega)\right)^{N}$. From Lemma 1 , as $X$ is closed in the weak topology of $\left(H_{0}^{1}(\Omega)\right)^{N}$, it follows that $\mathcal{E}$ is coercive in the weak topology of $X$.

Theorem 4 Let $(A 1)-(A 3)$ hold and let $S<4$. Then $\mathcal{E}$ possesses a minimizer in $X$.

Proof. This is an immediate consequence of Lemma 2 and 3 and the fundamental theorem of the calculus of variations, see, e. g., Dal Maso (1993); Struwe (1990).

The exchange and correlation functionals under LDA approximation satisfy the growth condition (A2) with $q=4 / 3$. Hence, the results of this section apply for the energy functional associated with KS-DFT. Theorem 4 establishes for $S \leq 3$ the existence of a self-consistent solution to the eigenvalue problem (Eqn. (35)), which determines the ground-state properties of a materials system.

\subsection{Convergence of the finite-element approximation}

Next we prove the convergence of finite-element approximation. We do so in two steps, first establishing the $\Gamma$-convergence of the restricted functional (Theorem 6 ), then its equicoercivity (Lemma 7). The main result is Theorem 8. By $\mathcal{P}_{k}$ we denote the ring of polynomials of non-negative degree less than or equal to $k$ for some fixed $k \geq 1$. Let $T_{h}$ be a family of triangulations of $\Omega$ of decreasing mesh size $h>0$, and let $X_{h}$ be the corresponding sequence of subspaces of $X$ consisting of functions whose restriction to every cell in $T_{h}$ is a polynomial, i. e.,

$X_{h}=\left\{\tilde{\Psi} \in\left(H_{0}^{1}(\Omega)\right)^{N}\left|\left\langle\tilde{\psi}_{i}, \tilde{\psi}_{j}\right\rangle_{\left(L^{2}(\Omega), L^{2}(\Omega)\right)}=\delta_{i j}, i, j=1,2, \ldots, N, \tilde{\psi}_{i}\right| T \in \mathcal{P}_{k}\right.$ for $\left.T \in T_{h}\right\}$.

Similarly, let

$$
X_{1_{h}}=\left\{\tilde{\phi} \in H_{0}^{1}(\Omega)|\tilde{\phi}| T \in \mathcal{P}_{k} \text { for } T \in T_{h}\right\}
$$

be the corresponding family of subspaces $X_{1_{h}}$ for the electrostatic problem. It follows from standard approximation theory that the sequence of spaces $X_{h}$ and $X_{1_{h}}$ become more and more dense in $X$ and $H_{0}^{1}(\Omega)$ as $h$ decreases. We define a sequence of discrete energy functionals

$$
\mathcal{E}_{h}(\Psi, \mathbf{R})= \begin{cases}\frac{1}{2}\|\nabla \Psi\|_{L^{2}(\Omega)}^{2}+G(\rho)+J_{h}(\rho, \mathbf{R}), & \text { if } \Psi \in X_{h} \\ +\infty, & \text { otherwise }\end{cases}
$$

where

$$
J_{h}(\rho, \mathbf{R})=-\min _{\phi \in H_{0}^{1}(\Omega)} I_{h}(\phi, \rho, \mathbf{R})
$$

and 


$$
I_{h}(\phi, \rho, \mathbf{R})= \begin{cases}I(\phi, \rho, \mathbf{R}) & \text { if } \phi \in X_{1_{h}}, \Psi \in X_{h}, \\ +\infty, & \text { otherwise, }\end{cases}
$$

where

$$
I(\phi, \rho, \mathbf{R})=\frac{1}{C_{S}} \int_{\Omega}|\nabla \phi(\mathbf{x})|^{2} \mathrm{~d} \mathbf{x}-\int_{\Omega}(\rho(\mathbf{x})+b(\mathbf{x}, \mathbf{R})) \phi(\mathbf{x}, \mathbf{R}) \mathrm{d} \mathbf{x}
$$

The following remark, see Gavini et al. (2007b), is needed before proceeding to the proof of $\Gamma$-convergence of the finite-element approximation.

Remark 5 If $\left(\Psi_{h}\right)_{h} \subset\left(X_{h}\right)$ is a sequence such that $\rho_{h} \rightarrow \rho$ in $L^{2}(\Omega)$, then

$$
\lim _{h \rightarrow 0} J_{h}\left(\rho_{h}\right)=J(\rho)
$$

Theorem 6 Let (A1), (A2) hold and let $S<4$. Then $\mathcal{E}_{h} \rightarrow \mathcal{E}$ (in the $\Gamma$-sense) in the weak topology of $X$.

Proof. To establish the $\Gamma$-convergence of the sequence of energy functionals we must establish the lim-inf inequality and construct a recovery sequence.

We first show the lim-inf inequality. Consider a sequence $\left(\Psi_{h}\right)_{h>0}$ such that $\Psi_{h} \rightarrow \Psi$ in $X$ as $h \searrow 0$. We only have to discuss the case that there exists a subsequence $\left(\Psi_{h_{k}}\right)_{k}$ with $\Psi_{h_{k}} \in X_{h_{k}}$ for every $k \in \mathbb{N}$. Otherwise the lim-inf inequality holds trivially,

$$
+\infty=\liminf _{k \rightarrow \infty} \mathcal{E}_{h_{k}}\left(\Psi_{h_{k}}, \mathbf{R}\right) \geq \mathcal{E}(\Psi, \mathbf{R}) .
$$

By (A1), (A2) and since $S<4$, it follows as in the proof of Lemma 2 that $\rho_{h_{k}} \rightarrow \rho$ in $L^{\max \{2, q\}}(\Omega)$ for $k \rightarrow \infty$. As $\mathcal{E}_{h}(\Psi, \mathbf{R}) \geq \frac{1}{2}\|\nabla \Psi\|_{L^{2}(\Omega)}^{2}+G(\rho)+J_{h}(\rho, \mathbf{R})$, we find

$$
\liminf _{k \rightarrow \infty} \mathcal{E}_{h_{k}}\left(\Psi_{h_{k}}, \mathbf{R}\right) \geq \liminf _{k \rightarrow \infty}\left\{\frac{1}{2}\left\|\nabla \Psi_{h_{k}}\right\|_{L^{2}(\Omega)}^{2}+G\left(\rho_{h_{k}}\right)+J_{h_{k}}\left(\rho_{h_{k}}, \mathbf{R}\right)\right\} .
$$

From the compact embeddings of $X$ into $L^{2}(\Omega)$ and $L^{q}(\Omega)$, Remark 5 which establishes the continuity of the discrete electrostatic problem in $L^{2}(\Omega)$, the continuity of $G$ in $L^{q}(\Omega)$ from (c), and the lower semi-continuity of $\left\|\nabla \Psi_{h}\right\|_{L^{2}(\Omega)}^{2}$ in the weak topology of $X$ from (d), it follows that

$$
\liminf _{k \rightarrow \infty} \mathcal{E}_{h_{k}}\left(\Psi_{h_{k}}, \mathbf{R}\right) \geq \mathcal{E}(\Psi, \mathbf{R})
$$

This establishes the lim-inf inequality.

The construction of a recovery sequence is trivial from the density of the finite-element approximation spaces in $H^{1}(\Omega)$ : Let $\left(\Psi_{h}\right)_{h>0}$ be a sequence constructed from the interpolation functions of successive triangulations such that $\Psi_{h} \rightarrow \Psi$ in $X$. From the continuity of individual terms of the energy functional, including the discrete electrostatic interaction energy from Remark 5, we have $\lim _{h \rightarrow 0} \mathcal{E}_{h}\left(\Psi_{h}, \mathbf{R}\right)=\mathcal{E}(\Psi, \mathbf{R})$. Hence we conclude $\mathcal{E}_{h} \rightarrow \mathcal{E}$ in the weak topology of $X$. 
Lemma 7 Let the assumptions (A2), (A3) hold. Then the family $\left(\mathcal{E}_{h}\right)_{h>0}$ is equi-coercive in the weak topology of $X$.

Proof. If $\Psi \notin X_{h}$, then $\mathcal{E}_{h}(\Psi, \mathbf{R})=+\infty$. If $\Psi \in X_{h}$, then $\mathcal{E}_{h}(\Psi, \mathbf{R})=\frac{1}{2}\|\nabla \Psi\|_{L^{2}(\Omega)}^{2}+$ $G(\rho)+J_{h}(\rho, \mathbf{R})$. Also, as the finite-element subspaces $X_{h}$ and $X_{1_{h}}$ are constructed from a single triangulation, this allows us to use each component of $\Psi \in X_{h}$ as a trial function in the electrostatic problem as demonstrated in Lemma 3 and Eqn. (42). Consequently, we arrive at the inequality

$$
J_{h}(\rho) \geq C_{0}\|\Psi\|_{L^{3}(\Omega)}^{3}-\frac{C_{0}}{C_{S}}\|\nabla \Psi\|_{L^{2}(\Omega)}^{2}-C\|\Psi\|_{L^{2}(\Omega)}^{2}
$$

for arbitrary $C_{0}>0$. Using the growth results for $G$, we have uniformly in $h$

$$
\mathcal{E}_{h}(\Psi) \geq \frac{1}{2}\left(1-\frac{2 C_{0}}{C_{S}}\right)\|\nabla \Psi\|_{L^{2}(\Omega)}^{2}+C_{0}\|\Psi\|_{L^{3}(\Omega)}^{3}-c_{0}\|\Psi\|_{L^{2 q}(\Omega)}^{2 q}-C\|\Psi\|_{L^{2}(\Omega)}^{2}-c_{1} .
$$

Choosing $0<2 C_{0}<C_{S}$ and since $2 q<3$, the expression on the right hand side is a coercive function independent of $h$ in the weak topology of $X$. Thus it follows that $\mathcal{E}_{h}$ is equi-coercive in the weak topology of $X$.

Theorem 8 Let the hypotheses (A1)-(A3) hold and let $S<4$. Then

$$
\lim _{h \rightarrow 0} \inf _{X} \mathcal{E}_{h}=\min _{X} \mathcal{E} .
$$

Proof. This result follows from Theorem 6, Lemma 7 and Dal Maso (1993, Theorem 7.8).

Remark 9 Theorem 8 establishes rigorously the convergence of the ground state energy of a system computed with a finite-element approximation. Additionally, if the ground state is not degenerate, the convergence of the ground-state electron density in $H_{0}^{1}(\Omega)$ follows.

\subsection{Convergence of the finite-element approximation with numerical quadratures}

An efficient implementation of the integrals in the functional requires a further approximation by numerical quadratures. In this section, we prove the convergence of the finiteelement approximation with numerical quadratures. We do so by combining ideas introduced in the previous section with well-known estimates of quadrature errors (Ciarlet (2002)).

Let $I=\int_{\Omega} f(\mathbf{x}) \mathrm{d} \mathbf{x}$ and if $\tilde{I}$ denotes a $n$-th order quadrature of $I$, the error associated with the numerical quadrature satisfies a bound of the type

$$
|\tilde{I}-I| \leq C_{\Omega}^{n+1} \int_{\Omega}\left|D^{n+1} f(\mathbf{x})\right| \mathrm{d} \mathbf{x}
$$


where $C_{\Omega}$ is a Poincare-type constant related to the size of the domain. Let the degree of the polynomial used in the finite-element interpolation be $k$ and identify $h$ with the maximum element size in the mesh. We define the energy functional where all integrations are performed with numerical quadrature rules (with $Q\{\mathcal{E}\} \equiv \tilde{\mathcal{E}}$ being the quadrature of $\mathcal{E})$ as

$$
\tilde{\mathcal{E}}_{h}(\Psi, \mathbf{R})= \begin{cases}Q\left\{\frac{1}{2}\|\nabla \Psi\|_{L^{2}(\Omega)}^{2}\right\}+\tilde{G}(\rho)+\tilde{J}_{h}(\rho, \mathbf{R}), & \text { if } \Psi \in X_{h}, \\ +\infty, & \text { otherwise }\end{cases}
$$

Here

$$
\tilde{J}_{h}(\rho, \mathbf{R})=-\min _{\phi \in H_{0}^{1}(\Omega)} \tilde{I}_{h}(\phi, \rho, \mathbf{R})
$$

and

$$
\tilde{I}_{h}(\phi, \rho, \mathbf{R})= \begin{cases}\tilde{I}(\phi, \rho, \mathbf{R})=Q\{I(\phi, \rho, \mathbf{R})\}, & \text { if } \phi \in X_{1_{h}}, \Psi \in X_{h} \\ +\infty, & \text { otherwise }\end{cases}
$$

The error in the energy functional introduced by the numerical quadrature satisfies the estimate

$$
\begin{aligned}
\left|\tilde{\mathcal{E}}_{h}(\Psi, \mathbf{R})-\mathcal{E}_{h}(\Psi, \mathbf{R})\right| \leq & C h^{n+1} \sum_{i} \int_{e_{i}}\left|D^{n+1}\left[\sum_{j=1}^{N}\left|\nabla \psi_{j}(\mathbf{x})\right|^{2}+g(\rho)\right]\right| \mathrm{d} \mathbf{x} \\
& +\left|\tilde{J}_{h}(\rho, \mathbf{R})-J_{h}(\rho, \mathbf{R})\right|
\end{aligned}
$$

where $e_{i}$ denotes the $i$-th element in the finite-element mesh. For $\Psi \in X_{h},\left|\nabla \psi_{j}(\mathbf{x})\right|$ is a polynomial function of degree $2(k-1)$ for $j=1,2, \ldots, N$. Thus, if $n-2(k-1) \geq 0$, then $D^{n+1}\left[\sum_{j=1}^{N}\left|\nabla \psi_{j}\right|^{2}\right]=0$. An application of the inverse inequality (Ciarlet (2002)) then gives the alternative estimate

$$
\begin{aligned}
\left|\tilde{\mathcal{E}}_{h}(\Psi, \mathbf{R})-\mathcal{E}_{h}(\Psi, \mathbf{R})\right| & \leq C h \sum_{i} \int_{e_{i}} \sum_{j=1}^{N}\left|2 g^{\prime}(\rho) \psi_{j}(\mathbf{x}) \nabla \psi_{j}(\mathbf{x})\right| \mathrm{d} \mathbf{x}+\left|\tilde{J}_{h}(\rho, \mathbf{R})-J_{h}(\rho, \mathbf{R})\right| \\
& \leq C h\left\|4\left(g^{\prime}(\rho)\right)^{2} \rho\right\|_{L^{1}(\Omega)}^{1 / 2}\|\nabla \Psi\|_{L^{2}(\Omega)}+\left|\tilde{J}_{h}(\rho, \mathbf{R})-J_{h}(\rho, \mathbf{R})\right| . \quad \text { (55) }
\end{aligned}
$$

Remark 10 If $\left(\Psi_{h}\right)_{h} \subset\left(X_{h}\right)$ is a sequence such that $\Psi_{h} \rightarrow \Psi$ in $X$ for $h \searrow 0$, if $n-2 k+3>0$ and $S<4$, then $\lim _{h \rightarrow 0} \inf _{H_{0}^{1}(\Omega)} \tilde{I}_{h}\left(\cdot, \rho_{h}, \mathbf{R}\right)=\min _{H_{0}^{1}(\Omega)} I(\cdot, \rho, \mathbf{R})$, i. e., $\lim _{h \rightarrow 0} \tilde{J}_{h}\left(\rho_{h}, \mathbf{R}\right)=J(\rho, \mathbf{R})($ Gavini et al. (2007b)).

We denote by hypothesis $(\mathrm{H})$ the following three assumptions:

(H.i) If $\left(\Psi_{h}\right)_{h} \subset\left(X_{h}\right)$ with $\Psi_{h} \rightarrow \Psi$ in $X$, then $\left\|\left(g^{\prime}\left(\rho_{h}\right)\right)^{2} \rho_{h}\right\|_{L^{1}(\Omega)}^{1 / 2}$ is bounded independently of $h$. 
(H.ii) $S<4$.

(H.iii) $n-2 k+3>0$.

Remark 11 If hypotheses $(H)$ hold, then it follows from Eqn. (55), Remark 5 and Remark 10 that $\lim _{h \rightarrow 0}\left\{\tilde{\mathcal{E}}_{h}\left(\Psi_{h}, \mathbf{R}\right)-\mathcal{E}_{h}\left(\Psi_{h}, \mathbf{R}\right)\right\}=0$.

We now turn to the convergence of finite-element approximations with numerical quadratures.

Theorem 12 Let (A1), (A2) and (H) hold. Then $\tilde{\mathcal{E}}_{h} \Gamma$-converges to $\mathcal{E}$ as $h \rightarrow 0$ in the weak topology of $X$.

Proof. Let $\left(\Psi_{h}\right)_{h}$ be a sequence such that $\Psi_{h} \rightarrow \Psi$. Without loss of generality we may assume the existence of a subsequence $\left(\Psi_{h_{k}}\right)_{k} \subset\left(\Psi_{h}\right)_{h}$ with $\Psi_{h_{k}} \in X_{h_{k}}$ for every $k$, as otherwise it trivially holds

$$
+\infty=\liminf _{k \rightarrow \infty} \tilde{\mathcal{E}}_{h_{k}}\left(\Psi_{h_{k}}, \mathbf{R}\right) \geq \mathcal{E}(\Psi, \mathbf{R}) .
$$

We observe

$$
\liminf _{k \rightarrow \infty} \tilde{\mathcal{E}}_{h_{k}}\left(\Psi_{h_{k}}, \mathbf{R}\right) \geq \liminf _{k \rightarrow \infty} \mathcal{E}_{h_{k}}\left(\Psi_{h_{k}}, \mathbf{R}\right)+\liminf _{k \rightarrow \infty}\left(\tilde{\mathcal{E}}_{h_{k}}-\mathcal{E}_{h_{k}}\right)\left(\Psi_{h_{k}}, \mathbf{R}\right) .
$$

It follows from Remark 10 that $\lim _{k \rightarrow \infty}\left(\tilde{\mathcal{E}}_{h_{k}}-\mathcal{E}_{h_{k}}\right)\left(\Psi_{h_{k}}, \mathbf{R}\right)=0$. Consequently, from Theorem 6

$$
\liminf _{k \rightarrow \infty} \tilde{\mathcal{E}}_{h_{k}}\left(\Psi_{h_{k}}, \mathbf{R}\right) \geq \liminf _{k \rightarrow \infty} \mathcal{E}_{h_{k}}\left(\Psi_{h_{k}}, \mathbf{R}\right) \geq \mathcal{E}(\Psi, \mathbf{R}) .
$$

This establishes the lim-inf inequality.

The construction of a recovery sequence is again trivial from the density of the finiteelement approximation spaces in $H^{1}(\Omega)$. Let $\left(\Psi_{h}\right)_{h>0}$ be a sequence constructed from the interpolation functions of successive triangulations such that $\Psi_{h} \rightarrow \Psi$ in $X$. From Eqn. (55) we have

$$
\lim _{h \rightarrow 0} \tilde{\mathcal{E}}_{h}\left(\Psi_{h}, \mathbf{R}\right)=\lim _{h \rightarrow 0} \mathcal{E}_{h}\left(\Psi_{h}, \mathbf{R}\right)=\mathcal{E}(\Psi, \mathbf{R})
$$

Hence, we conclude $\tilde{\mathcal{E}}_{h} \rightarrow \mathcal{E}$ in the weak topology of $X$.

Lemma 13 If (A3) and $(H)$ hold, then the energy functional $\tilde{\mathcal{E}}_{h}$ obtained by numerical quadrature is equi-coercive in the weak topology of $X$.

Proof. We observe from Eqn. (45) that if $\Psi \in X_{h}$,

$$
\mathcal{E}_{h}(\Psi, \mathbf{R}) \geq \frac{1}{2}\left(1-\frac{2 C_{0}}{C_{S}}\right)\|\nabla \Psi\|_{L^{2}(\Omega)}^{2}+C_{0}\|\Psi\|_{L^{3}(\Omega)}^{3}-c_{0}\|\Psi\|_{L^{2 q}(\Omega)}^{2 q}-C\|\Psi\|_{L^{2}(\Omega)}^{2}-c_{1} .
$$

Since $2 q<3$, there exist positive constants $C_{1}, C_{2}$ and $C_{3}$ such that

$$
\mathcal{E}_{h}(\Psi, \mathbf{R}) \geq C_{1}\|\nabla \Psi\|_{L^{2}(\Omega)}^{2}+C_{2}\|\Psi\|_{L^{2}(\Omega)}^{2}-C_{3} .
$$


This implies

$$
\tilde{\mathcal{E}}_{h}(\Psi, \mathbf{R}) \geq C_{1}\|\nabla \Psi\|_{L^{2}(\Omega)}^{2}+C_{2}\|\Psi\|_{L^{2}(\Omega)}^{2}-C_{3}-C h\|\Psi\|_{L^{2}(\Omega)}\|\nabla \Psi\|_{L^{2}(\Omega)} .
$$

So there exists a bound $\bar{h}>0$ such that for all $h<\bar{h}$

$$
\mathcal{E}_{h}(\Psi, \mathbf{R}) \geq K_{0}\|\nabla \Psi\|_{L^{2}(\Omega)}^{2}+K_{1}\|\Psi\|_{L^{2}(\Omega)}^{2}-K_{2},
$$

where $K_{0}, K_{1}$, and $K_{2}$ are positive constants independent of $h$. From this inequality it follows that $\tilde{\mathcal{E}}_{h}$ is equi-coercive in the weak topology of $X$.

Theorem 14 Let (A1)-(A3) and (H) hold. Then $\lim _{h \rightarrow 0} \inf _{X} \tilde{\mathcal{E}}_{h}=\min _{X} \mathcal{E}$.

Proof. This result follows again from Theorem 12, Lemma 13, and Dal Maso (1993, Theorem 7.8).

For the LDA approximation of exchange-correlation functionals, hypothesis (A1)-(A3) hold with $q=4 / 3$. Also if $S \leq 3$, it is straightforward to check that (H.i) holds. Choosing appropriate quadrature rules which satisfy $n-2 k+3>0$, the convergence of the self-consistent eigenvalue problem of KS-DFT using a finite-element approximation with numerical quadratures is established.

\subsection{Pseudopotential approximation}

The tightly bound core electrons are chemically inactive and hence have a negligible contribution towards determining physical properties such as binding energies and bond lengths. The core states are localized in the vicinity of the nucleus leading to oscillations of the valence wavefunctions in this region due to the orthogonality requirement. Regardless of the basis set used, a large number of basis functions is needed to capture these oscillations. This difficulty may be overcome by recourse to the pseudopotential approximation (Pickett (1989)) in which the all-electron potential $V_{\text {ext }}(\mathbf{x}, \mathbf{R})$ in Eqn. (29) is replaced by an effective potential $V_{\text {ext }}^{\mathrm{PS}}(\mathbf{x}, \mathbf{R})$ (or equivalently the energy $E_{\text {ext }}(\rho, \mathbf{R})$ in Eqn. (22) with an effective energy $\left.E_{\text {ext }}^{\mathrm{PS}}(\rho, \mathbf{R})\right)$ and the core (non-valence) electrons are eliminated. The idea is that these effective potentials describe the effect of the core electrons adequately and represent the valence electrons by means of nodeless pseudo-wavefunctions. This approximation enables the solution of much larger systems than could otherwise be analyzed.

Based on their spatial dependence, pseudopotentials can be broadly classified as local and non-local. The non-local pseudopotentials, which include norm conserving (Bachelet et al. (1982); Rappe et al. (1990); Troullier and Martins (1991)) and ultrasoft pseudopotentials (Vanderbilt (1990)), are angular momentum dependent and are designed to accurately reproduce the scattering properties of the all-electron potential, thereby ensuring greater accuracy and transferability. They are usually employed in the Kleinman-Bylander form 
(Kleinman and Bylander (1982)), which is less computationally expensive than the original semi-local form. In the sequel, we prove the existence of minimizers and convergence of the finite-element approximation with numerical quadratures for local as well as for non-local pseudopotential approximations.

\subsubsection{Local pseudopotential}

A local pseudopotential is an explicit function $V_{\text {ext }}^{\mathrm{PS}}(\mathbf{x}, \mathbf{R})$ with $\left\|V_{\mathrm{ext}}^{\mathrm{PS}}\right\|_{L^{\infty}} \leq C$. The local pseudopotential approximation can then be incorporated into our variational formulation by replacing $b(\mathbf{x}, \mathbf{R})$ with $b^{\mathrm{PS}}(\mathbf{x}, \mathbf{R})=-\frac{2}{C_{S}} \nabla^{2} V_{\text {ext }}^{\mathrm{PS}}(\mathbf{x}, \mathbf{R})$. Consequently, all the results presented in the previous sections are applicable and hence existence of a minimum and convergence of the finite-element approximation with numerical quadratures follow.

\subsubsection{Non-local pseudopotential}

A non-local pseudopotential is an operator on the wavefunction $\psi$. In the KleinmanBylander form it is expressed for $J \in\{1,2, \ldots, M\}$ as

$$
V_{\mathrm{ion}}^{J}\left(\mathbf{x}, \mathbf{R}_{J}\right) \psi(\mathbf{x})=V_{\mathrm{loc}}^{J}\left(\mathbf{x}, \mathbf{R}_{J}\right) \psi(\mathbf{x})+\sum_{l m} C_{l m}^{J} u_{l m}^{J}\left(\mathbf{x}, \mathbf{R}_{J}\right) \Delta V_{l}^{J}\left(\mathbf{x}, \mathbf{R}_{J}\right),
$$

where

$$
\begin{aligned}
\Delta V_{l}^{J}\left(\mathbf{x}, \mathbf{R}_{J}\right) & =V_{l}^{J}\left(\mathbf{x}, \mathbf{R}_{J}\right)-V_{\mathrm{loc}}^{J}\left(\mathbf{x}, \mathbf{R}_{J}\right) \\
C_{l m}^{J} & =\frac{\int_{\Omega} u_{l m}^{J}\left(\mathbf{x}, \mathbf{R}_{J}\right) \Delta V_{l}^{J}\left(\mathbf{x}, \mathbf{R}_{J}\right) \psi(\mathbf{x}) \mathrm{d} \mathbf{x}}{\int_{\Omega} u_{l m}^{J}\left(\mathbf{x}, \mathbf{R}_{J}\right) \Delta V_{l}^{J}\left(\mathbf{x}, \mathbf{R}_{J}\right) u_{l m}^{J}\left(\mathbf{x}, \mathbf{R}_{J}\right) \mathrm{d} \mathbf{x}} .
\end{aligned}
$$

$V_{l}^{J}\left(\mathbf{x}, \mathbf{R}_{J}\right)$ is the ionic pseudopotential component corresponding to the azimuthal quantum number $l, V_{\text {loc }}^{J}\left(\mathbf{x}, \mathbf{R}_{J}\right)$ is the local ionic potential and $u_{l m}^{J}\left(\mathbf{x}, \mathbf{R}_{J}\right)$ represents the pseudo-wavefunction for the valence states of interest, all for a single atom. The superscript $J$ is for the atom number and the subscript $m$ denotes the magnetic quantum number. Therefore

$$
V_{\mathrm{ext}}^{P S}(\mathbf{x}, \mathbf{R}) \psi(\mathbf{x})=\sum_{J=1}^{M} V_{\mathrm{loc}}^{J}\left(\mathbf{x}, \mathbf{R}_{J}\right) \psi(\mathbf{x})+\sum_{J=1}^{M} \sum_{l m} C_{l m}^{J} u_{l m}^{J}\left(\mathbf{x}, \mathbf{R}_{J}\right) \Delta V_{l}^{J}\left(\mathbf{x}, \mathbf{R}_{J}\right) .
$$

Let us redefine $V_{\text {ext }}(\mathbf{x}, \mathbf{R})=\sum_{J=1}^{M} V_{\text {loc }}^{J}\left(\mathbf{x}, \mathbf{R}_{J}\right)$, for which we can obtain the corresponding $b(\mathbf{x}, \mathbf{R})$. The energy functional for the pseudopotential can therefore be written as

$$
\mathcal{E}^{P S}(\Psi, \mathbf{R})=\mathcal{E}(\Psi, \mathbf{R})+\mathcal{K}(\Psi, \mathbf{R})
$$

where 


$$
\begin{aligned}
\mathcal{K}(\Psi, \mathbf{R}) & =\sum_{i=1}^{N_{v}} \sum_{J=1}^{M} \sum_{l m} \frac{1}{G_{l m}^{J}}\left|\int_{\Omega} f\left(\mathbf{x}, \mathbf{R}_{J}\right) \psi_{i}(\mathbf{x}) \mathrm{d} \mathbf{x}\right|^{2}, \\
f\left(\mathbf{x}, \mathbf{R}_{J}\right) & =u_{l m}^{J}\left(\mathbf{x}, \mathbf{R}_{J}\right) \Delta V_{l}^{J}\left(\mathbf{x}, \mathbf{R}_{J}\right), \\
G_{l m}^{J} & =\int_{\Omega} u_{l m}^{J}\left(\mathbf{x}, \mathbf{R}_{J}\right) \Delta V_{l}^{J}\left(\mathbf{x}, \mathbf{R}_{J}\right) u_{l m}^{J}\left(\mathbf{x}, \mathbf{R}_{J}\right) \mathrm{d} \mathbf{x} .
\end{aligned}
$$

$N_{v}$ is the total number of electrons after the pseudopotential approximation, i. e., valence electrons.

We make the assumption that $f \in H_{0}^{1}(\Omega)$ and $V_{\text {loc }}^{J} \in L^{\infty}(\Omega)$. Since $V_{\text {loc }}^{J}$ is in principle arbitrary, it can be always chosen such that $G_{l m}^{J} \neq 0$. We also note the following properties:

(B1) The functional $\mathcal{K}$ is continuous in $L^{2}(\Omega)$.

This property can be directly verified using Hölder's inequality.

(B2) $\mathcal{K} \geq-C_{1}\|\Psi\|_{L^{2}(\Omega)}^{2}$, where $C_{1}$ is a constant dependent on $f, G_{l m}^{J}$.

This inequality can immediately be obtained using Hölder's inequality.

Theorem 15 Let $(A 1)-(A 3)$ hold and let $S<4$. Then $\mathcal{E}^{P S}(\Psi, \mathbf{R})$ possesses a minimizer in $X$.

Proof. The lower semi-continuity of $\mathcal{E}^{P S}(\Psi, \mathbf{R})$ in the weak topology of $X$ follows from Lemma 2 and (B1). From Lemma 3 and (B2) it is clear that $\mathcal{E}^{P S}(\Psi, \mathbf{R})$ is coercive in the weak topology of $X$. Therefore from the fundamental theorem of the calculus of variations, see, e. g., Dal Maso (1993), $\mathcal{E}^{P S}(\Psi, \mathbf{R})$ has a minimizer in $X$.

Theorem 16 Let the hypotheses (A1)-(A3) hold and let $S<4$. Then

$$
\lim _{h \rightarrow 0} \inf _{X} \mathcal{E}_{h}^{P S}=\min _{X} \mathcal{E}^{P S} .
$$

Proof. Using the procedure outlined in Theorem 6 along with (B1) gives $\mathcal{E}_{h}^{P S} \rightarrow \mathcal{E}^{P S}$ (in the $\Gamma$-sense). The equi-coercivity of $\left(\mathcal{E}_{h}^{P S}\right)_{h>0}$ follows from Lemma 7 and (B2). Hence $\lim _{h \rightarrow 0} \inf _{X} \mathcal{E}_{h}^{P S}=\min _{X} \mathcal{E}^{P S}$ (Dal Maso (1993, Theorem 7.8)).

Theorem 17 Let $(A 1)-(A 3)$ and $(H)$ hold. Then $\lim _{h \rightarrow 0} \inf _{X} \tilde{\mathcal{E}}_{h}^{P S}=\min _{X} \mathcal{E}^{P S}$.

Proof. Consider $I=\int_{\Omega} f_{1}(x) f_{2}(x) \mathrm{d} \mathbf{x}$ for $f_{1}, f_{2} \in H_{0}^{1}(\Omega)$. The error due to numerical quadrature is

$$
\begin{aligned}
|I-\tilde{I}| & \leq C_{0} h^{n+1} \sum_{i} \int_{e_{i}} D^{n+1}\left(f_{1} f_{2}\right) \mathrm{d} \mathbf{x} \\
& \leq C h\left[\left\|\nabla f_{1}\right\|_{L^{2}(\Omega)}\left\|f_{2}\right\|_{L^{2}(\Omega)}+\left\|f_{1}\right\|_{L^{2}(\Omega)}\left\|\nabla f_{2}\right\|_{L^{2}(\Omega)}\right] .
\end{aligned}
$$

The last inequality is obtained by using the inverse inequality (Ciarlet (2002)) and Hölder's inequality. Therefore we have $\lim _{h \rightarrow 0}\{I-\tilde{I}\}=0$. Hence it follows from Remark 11 that 
$\lim _{h \rightarrow 0}\left\{\tilde{\mathcal{E}}_{h}^{P S}\left(\Psi_{h}, \mathbf{R}\right)-\mathcal{E}_{h}^{P S}\left(\Psi_{h}, \mathbf{R}\right)\right\}=0$. Adopting the same procedure as in Theorem 12 we obtain $\tilde{\mathcal{E}}_{h}^{P S} \rightarrow \mathcal{E}^{P S}$ (in the $\Gamma$-sense). Using (B2) in Lemma 13 gives the equi-coercivity of $\tilde{\mathcal{E}}_{h}^{P S}$ in the weak topology of $X$. From Dal Maso (1993, Theorem 7.8) $\lim _{h \rightarrow 0} \inf _{X} \tilde{\mathcal{E}}_{h}^{P S}$ $=\min _{X} \mathcal{E}^{P S}$.

In this entire section, for notational simplicity we have not incorporated spin polarization into our analysis. Note however that this simplification has no bearing on the analysis/results presented here. In particular, it is clear that even with spin polarization, the required growth condition given by (A2) still holds, ensuring that all the results are applicable.

\section{Numerical implementation}

We now turn to the numerical implementation of the variational formulation described in Section 2. The variational problem (Eqn. (20)) is discretized using the finite-element interpolation scheme

$$
\begin{aligned}
\psi_{i \sigma}(\mathbf{x}) & =\sum_{j=1}^{N_{h}} \psi_{i \sigma}^{j} N_{j}(\mathbf{x}), \\
\phi(\mathbf{x}) & =\sum_{j=1}^{N_{h}} \phi^{j} N_{j}(\mathbf{x})
\end{aligned}
$$

to obtain

$$
\begin{array}{r}
\sum_{j=1}^{N_{h}}\left[\int_{\Omega}\left(\frac{1}{2} \nabla N_{j}(\mathbf{x}) \nabla N_{k}(\mathbf{x})+V_{h, \text { eff }}^{\sigma}(\mathbf{x}, \mathbf{R}) N_{j}(\mathbf{x}) N_{k}(\mathbf{x})\right) \mathrm{d} \mathbf{x}\right] \psi_{i \sigma}^{j} \\
=\sum_{q=1}^{N_{\sigma}} \sum_{j=1}^{N_{h}} \lambda_{i q}^{\sigma} \psi_{q \sigma}^{j} \int_{\Omega} N_{j}(\mathbf{x}) N_{k}(\mathbf{x}) \mathrm{d} \mathbf{x} \\
\sum_{j=1}^{N_{h}}\left[\frac{1}{4 \pi} \int_{\Omega} \nabla N_{k}(\mathbf{x}) \nabla N_{j}(\mathbf{x}) \mathrm{d} \mathbf{x}\right] \phi^{j}=\int_{\Omega}\left(\rho_{h}(\mathbf{x})+b(\mathbf{x}, \mathbf{R})\right) N_{k}(\mathbf{x}) \mathrm{d} \mathbf{x} \\
\sum_{s=1}^{N_{h}} \sum_{r=1}^{N_{h}}\left[\int_{\Omega} N_{r}(\mathbf{x}) N_{s}(\mathbf{x}) \mathrm{d} \mathbf{x}\right] \psi_{m \sigma}^{r} \psi_{n \sigma}^{s}=\delta_{m n},
\end{array}
$$

where $\left(N_{k}\right)_{1 \leq k \leq N_{h}}$ are the basis functions of $X_{h}$, the Lagrange multipliers $\lambda_{i q}^{\sigma}$ are used to enforce the constraints (Eqn. 21), $V_{h \text {,eff }}^{\sigma}, \rho_{h}$ denote the discretized $V_{\text {eff }}^{\sigma}, \rho$ respectively, $\sigma \in\{\alpha, \beta\}$ and $i, m, n=1,2, \ldots, N_{\sigma}$. In solving the nonlinear system of Eqns. (73), (74) and (75), the Newton-Raphson method suggests itself as a means of achieving quadratic convergence in the vicinity of the solution. However, the Newton-Raphson algorithm is only conditionally convergent and great care must be exercised to ensure that the initial 
guess lies within the radius of convergence of the solution. In addition, the quadratic convergence rate is only asymptotic and the method may exhibit slow convergence during the early stages of iteration. In view of these limitations of the Newton-Raphson method, an iterative solution scheme based on the equivalent generalized nonlinear eigenvalue problem

$$
\mathbf{A}^{\sigma} \tilde{\Psi}^{\sigma}=\epsilon \mathbf{M} \tilde{\Psi}^{\sigma}, \quad \sigma \in\{\alpha, \beta\}
$$

may be advantageous early on in the iteration. Here,

$$
\begin{aligned}
\mathbf{A}^{\sigma}{ }_{i j} & =\frac{1}{2} \int_{\Omega} \nabla N_{i}(\mathbf{x}) \nabla N_{j}(\mathbf{x}) \mathrm{d} \mathbf{x}+\int_{\Omega} V_{\mathrm{h}, \mathrm{eff}}^{\sigma}(\mathbf{x}, \mathbf{R}) N_{i}(\mathbf{x}) N_{j}(\mathbf{x}) \mathrm{d} \mathbf{x}, \\
\mathbf{M}_{i j} & =\int_{\Omega} N_{i}(\mathbf{x}) N_{j}(\mathbf{x}) \mathrm{d} \mathbf{x},
\end{aligned}
$$

and $\tilde{\Psi}$ is a vector of the nodal values of the wavefunction. Evidently, Eqn. (76) needs to be solved self-consistently with Eqn. (74). In general, a large numbers of finite-element basis functions per atom is required for accuracy and convergence (Pask and Sterne (2005)), thus rendering the SCF method computationally expensive since it now entails the repeated solution of large systems of linear equations.

Keeping the above discussion in mind, we employ the following methodology. Since we expect the electron density to decay much more rapidly than the electrostatic potential, the wavefunctions are solved on a smaller domain $\Omega^{\Psi} \subset \Omega$. We employ zero Dirichlet boundary conditions for both the wavefunctions and the electrostatic potential on their respective domains. We first construct a coarse Delaunay triangulation $\mathcal{T}_{0}$ of the domain $\Omega$, with the nodes positioned at a coarsening rate of $r^{6 / 5}$ away from the nuclei, which is optimal for capturing the $1 / r$ decay with linear interpolation. Such a triangulation has high resolution in $\Omega^{\psi}$ and coarsens away rapidly in $\Omega \backslash \Omega^{\psi}$, cf. Fig. 1 for a single-atom example. In addition, all nuclei are located on nodes of this triangulation.

A solution procedure that combines the best attributes of the Newton-Raphson and the SCF methods may be devised as follows. We start by solving Eqns. (74) and (76) using the SCF method on $\mathcal{T}_{0}$. Specifically, we employ the implicitly restarted Lanczos method (Saad (1992)) for solving the linear eigenvalue problem and the conjugate gradient algorithm for the linear solver. We rewrite Eqn. (76) as

$$
\left(\mathbf{A}^{\sigma}-\eta \mathbf{M}\right)^{-1} \mathbf{M} \tilde{\Psi}^{\sigma}=\hat{\epsilon} \tilde{\Psi}^{\sigma}
$$

where $\eta$ is the shift parameter and $\hat{\epsilon}=\frac{1}{\epsilon-\eta}$. By choosing an appropriate value for $\eta$, we magnify the part of the eigenvalue spectrum of interest, thereby significantly increasing the rate of convergence of the eigenvalue solver without incurring any additional cost. Also, since $\mathcal{T}_{0}$ is very coarse, the computational expense involved for this step is minimal. Next, we apply Freudenthal's tetrahedron subdivision algorithm (Bey and Aachen (2000)) to $\mathcal{T}_{0}$ to obtain a finer triangulation $\mathcal{T}_{1}$ (Fig. 2) that is an uniform subdivision of $\mathcal{T}_{0}$. The preliminary solution obtained on $\mathcal{T}_{0}$ is transferred to $\mathcal{T}_{1}$ using the shape functions on $\mathcal{T}_{0}$. This transferred solution serves as a starting guess for the solution of Eqns. 
(73), (74) and (75), simultaneously using Newton's method with Goldstein-Armijo linesearches (Dennis and Schnabel (1996)). For the associated linear solver we use the Generalized Minimal Residual method (GMRES, Saad and Schultz (1986)) while retaining the option of switching to either the Bi-Conjugate Gradient Stable method (Bi-CGSTAB, van der Vorst (1992)) or Transpose-Free Quasi-Minimal Residual method (TFQMR, Freund (1993)). Since the Hessian information is required only through its product with a vector, we evaluate it by using directional derivative quotients. This approach is significantly faster than evaluating the exact Hessian. However, we also use the exact Hessian when required. The quality of the initial guess obtained by the procedure just described starts the Newton-Raphson iteration well within the radius of convergence, thereby ensuring convergence within exceedingly tight tolerances at a quadratic rate in no more than 6 or 7 iterations.

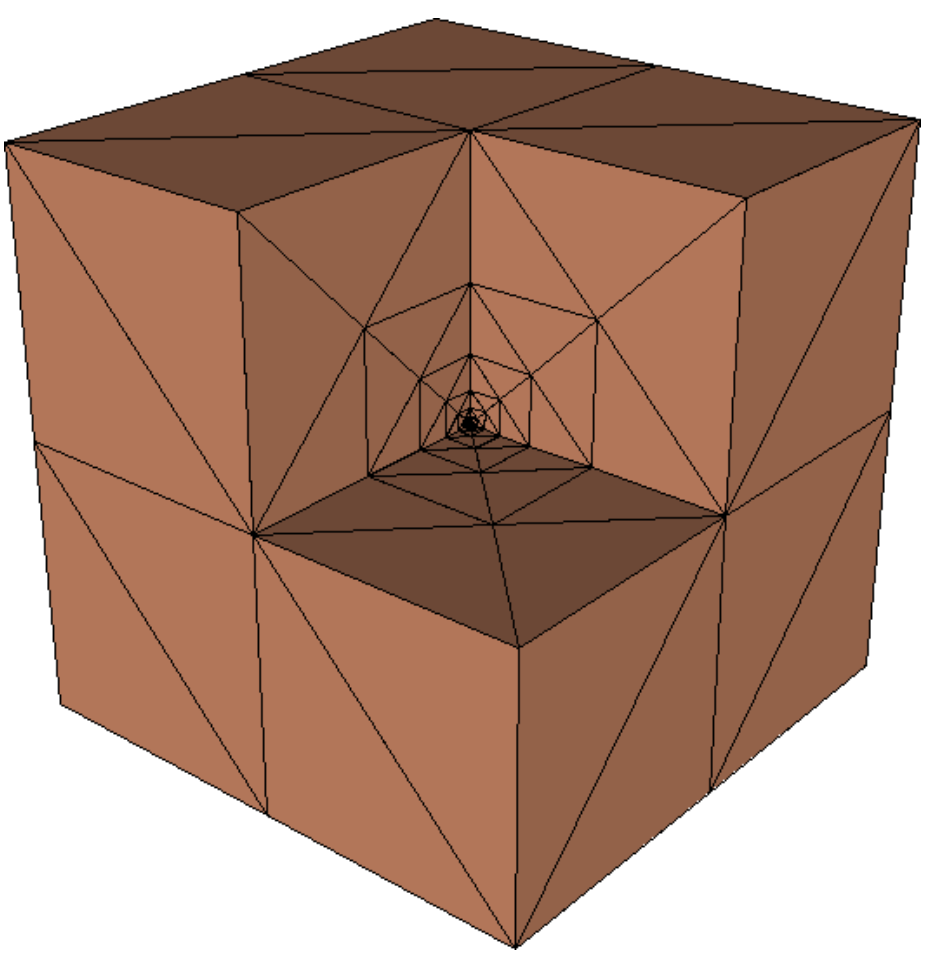

Fig. 1. Mesh of a sliced cubical domain corresponding to the triangulation $\mathcal{T}_{0}$. 


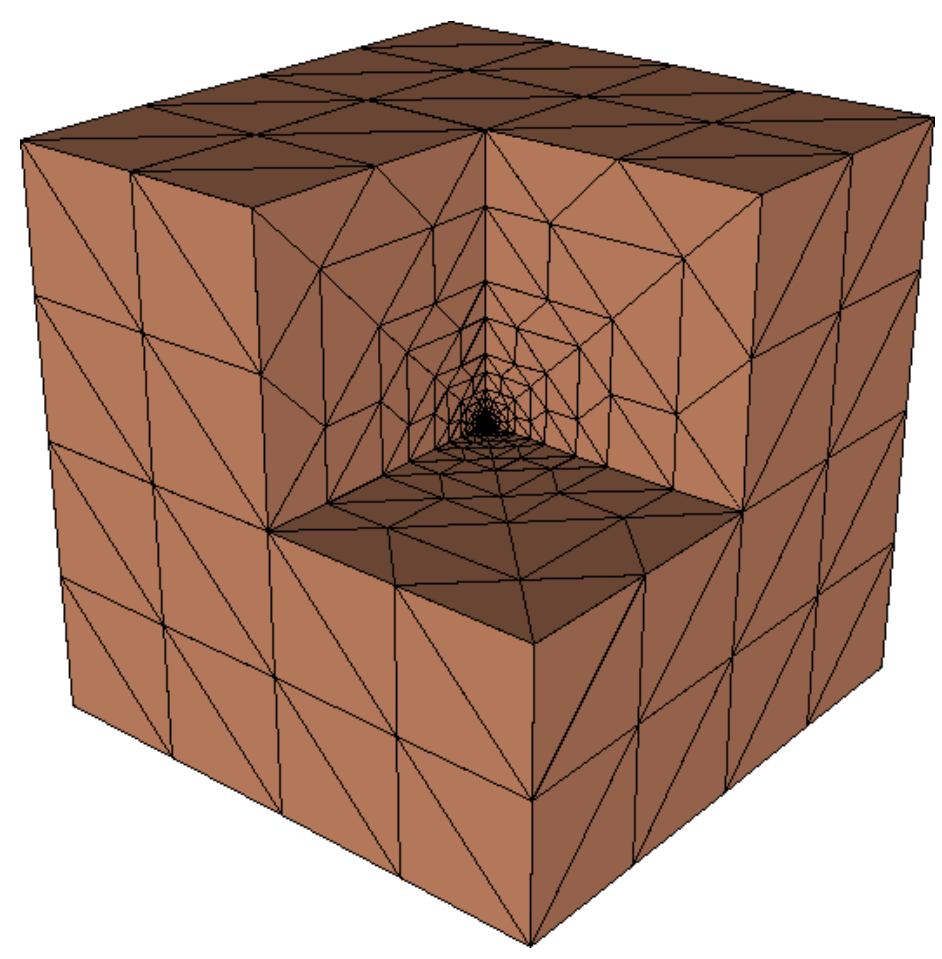

Fig. 2. Mesh of a sliced cubical domain corresponding to the triangulation $\mathcal{T}_{1}$.

The equilibrium position of the nuclei, defining an equilibrium configuration of the system, are computed using conjugate gradients with secant line search. The relevant forces to be equilibrated are derived in Appendix A. As the nuclei move, the triangulations must be updated. We do so simply by convecting the meshes according to the displacement field defined by the triangulation $\mathcal{T}_{0}$ on which the nuclei are located. The quality of these convected meshes is monitored throughout the calculations, and a complete remeshing is performed if the mesh quality falls below a prespecified tolerance. Each force update following a displacement of the nuclei requires the re-evaluation of the electrostatic potential and wavefunctions within an internal loop. In order to expedite this re-evaluation, we start from the initial electrostatic potential and wavefunctions that are convected from the previous configuration.

In order to verify convergence with respect to mesh size, we repeat the calculations on increasingly finer triangulations $\left\{\mathcal{T}_{n}\right\}$ obtained by successive uniform subdivisions. The solution on the parent triangulation is used as a starting guess on the finer triangulation, which ensures rapid convergence. This process is repeated until convergence is achieved to within a prespecified tolerance. The recursive nature of the calculations is advantageous with respect to solution schemes that require a complete restart every time the discretization is modified, e. g., plane wave basis calculations in which convergence has to be verified with respect to the energy cut-off and number of k-points sampled.

Finally, the formulation is implemented in parallel simply by recourse to domain decomposition. Owing to the local character of the majority of the calculations, domain decom- 
position may be expected to scale near-optimally. This expectation is indeed born out by numerical tests, cf. Section 5.4.

\section{Examples and results}

\section{1 'All-electron' calculations}

\subsubsection{Atoms}

The first set of examples considered are atoms of helium, lithium, carbon, nitrogen and oxygen. The triangulation $\mathcal{T}_{0}$ generated for these atoms, which has roughly 300 nodes, is depicted in Fig 1. The convergence of the ground state energy of the helium atom on increasing the subdivision number (i. e., number of applications of the subdivision algorithm) is shown in Fig 3. It is clear that there is rapid convergence of the energy on decreasing the mesh size. This is representative of the other examples. Table 1 shows that we reproduce the ground state energies obtained by the highly accurate calculations of Kotochigova et al. (1997). The deviation from experiment reflects the well-known deficiencies of LSDA.

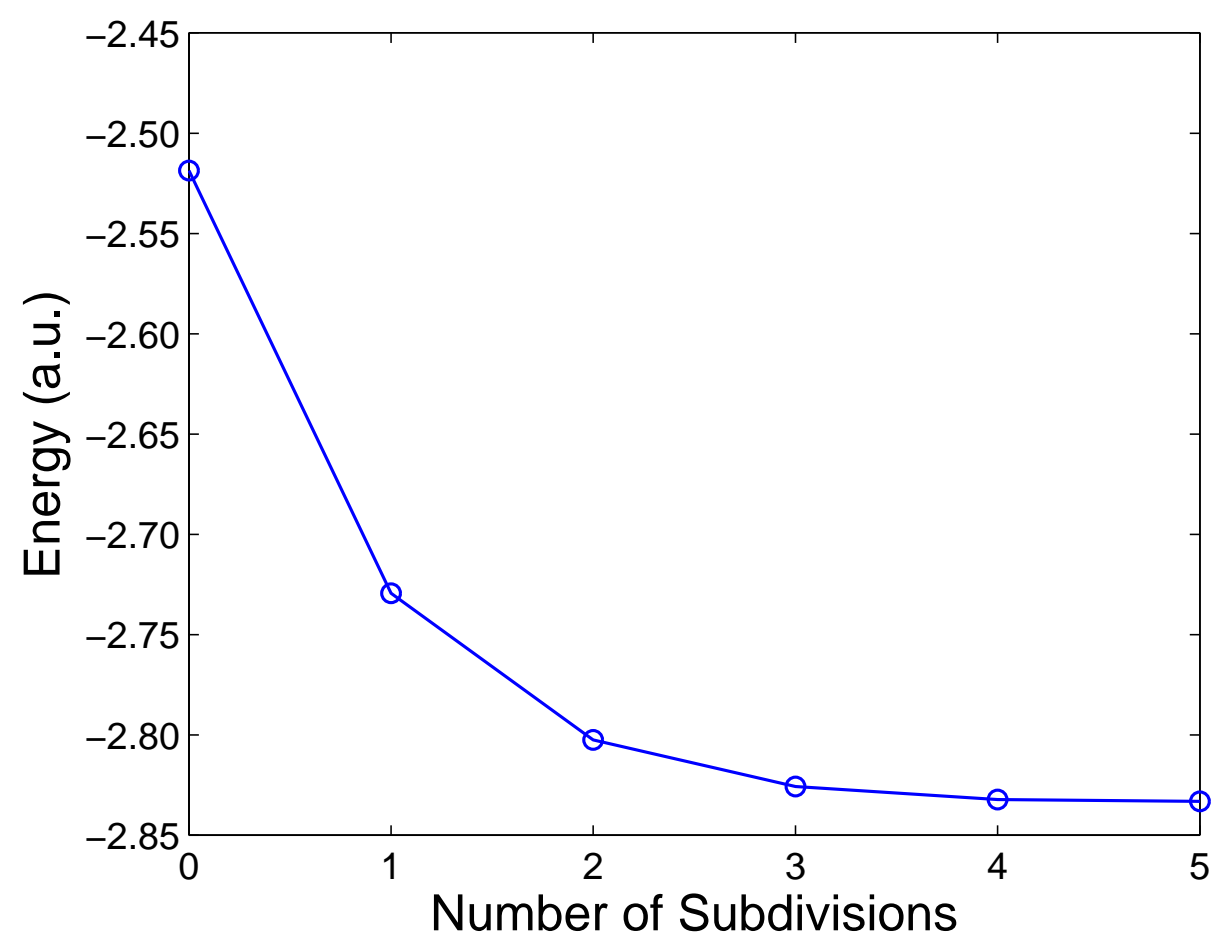

Fig. 3. Energy of the helium atom as a function of the number of uniform subdivisions of triangulation $\mathcal{T}_{0}$. 
Table 1

Ground state energies of selected atoms (a.u)

\begin{tabular}{ccccc}
\hline Element & KS-DFT-FE & KS-LSDA & KS-LSDA & $\begin{array}{c}\text { Experiments } \\
\end{array}$ \\
$\begin{array}{ccccc}\text { (This work) } \\
\text { He }\end{array}$ & -2.833 & -2.834 & -2.833 & -2.904 \\
$\mathrm{Li}$ & -7.340 & -7.343 & -7.343 & -7.478 \\
$\mathrm{C}$ & -37.460 & -37.470 & -37.466 & -37.844 \\
$\mathrm{~N}$ & -54.125 & -54.136 & -54.129 & -54.587 \\
$\mathrm{O}$ & -74.518 & -74.527 & -74.521 & -75.063 \\
\hline
\end{tabular}

\subsubsection{Molecules}

The next set of examples are nitrogen $\left(\mathrm{N}_{2}\right)$ and carbon monoxide $(\mathrm{CO})$ molecules. Tables 2 and 3 show that the binding energy and bond length that we obtain are in very good agreement with values in literature computed using plane waves (Engel et al. (2001)). Again, it is well known that LSDA predicts over-binding, i. e., higher binding energies and smaller bond lengths compared to experiments. The occupied valence molecular orbitals of CO can be seen in Fig. 4.

Table 2

Binding energy and bond length of $\mathrm{N}_{2}$

\begin{tabular}{ccccc}
\hline Property & $\begin{array}{c}\text { KS-DFT-FE } \\
\text { (This work) }\end{array}$ & $\begin{array}{c}\text { KS-LSDA } \\
\text { (Engel et al. (2001)) }\end{array}$ & $\begin{array}{c}\text { OFDFT-FE } \\
\text { (Gavini et al. (2007b)) }\end{array}$ & $\begin{array}{c}\text { Experiments } \\
\text { (Huber (1972)) }\end{array}$ \\
\hline Binding energy (eV) & -11.6 & -11.593 & -11.9 & -9.81 \\
Bond length (a.u.) & 2.06 & 2.068 & 2.7 & 2.07 \\
\hline
\end{tabular}

Table 3

Binding energy and bond length of $\mathrm{CO}$

\begin{tabular}{ccccc}
\hline Property & $\begin{array}{c}\text { KS-DFT-FE } \\
\text { (This work) }\end{array}$ & $\begin{array}{c}\text { KS-LSDA } \\
\text { (Engel et al. (2001)) }\end{array}$ & $\begin{array}{c}\text { OFDFT-FE } \\
\text { (Gavini et al. (2007b)) }\end{array}$ & $\begin{array}{c}\text { Experiments } \\
\text { (Huber (1972)) }\end{array}$ \\
\hline Binding energy (eV) & -13.03 & -12.967 & -12.6 & -11.2 \\
Bond length (a.u.) & 2.08 & 2.128 & 2.75 & 2.13 \\
\hline
\end{tabular}



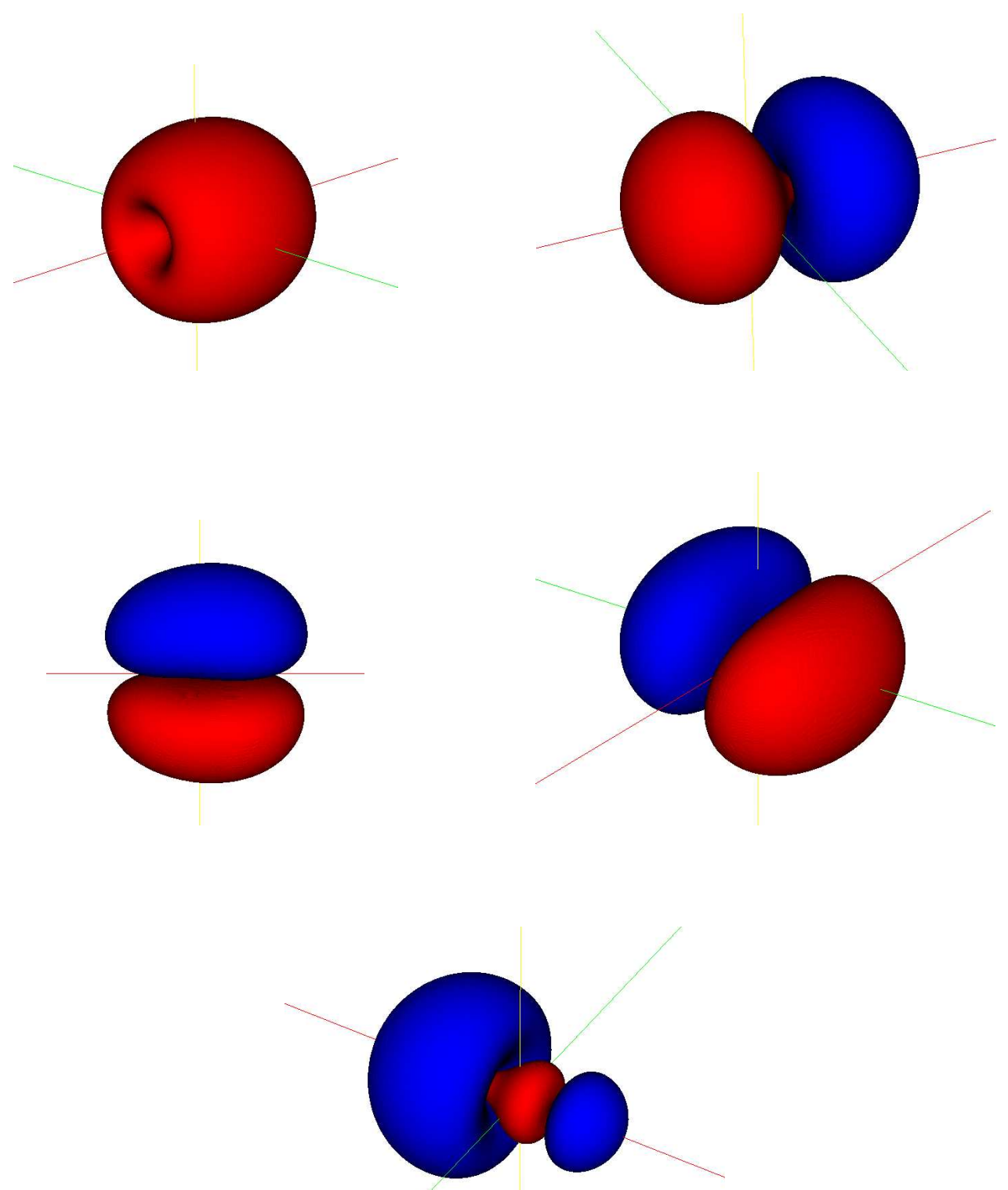

Fig. 4. Occupied valence molecular orbitals of CO.

\subsection{Pseudopotential approximation (local)}

In this section we present the results obtained using the smooth local 'Evanescent Core' pseudopotential (Fiolhais et al. (1995)). This pseudopotential has the form

$$
V_{\text {ion }}^{I}\left(\mathbf{x}, \mathbf{R}_{I}\right)=-\frac{Z}{R_{c}}\left\{\frac{1}{y}\left(1-(1+\beta y) e^{-\alpha y}\right)-A e^{-y}\right\},
$$

where $Z$ is the number of valence electrons and $y=\left|\mathbf{x}-\mathbf{R}_{I}\right| / R_{c}$. The core decay length $R_{c}$ and $\alpha \geq 0$ are element-dependent constants whose values can be obtained 
from Fiolhais et al. (1995). The relations used to evaluate $\beta$ and $A$ are

$$
\beta=\frac{\alpha^{3}-2 \alpha}{4\left(\alpha^{2}-1\right)}, \quad A=\frac{1}{2} \alpha^{2}-\alpha \beta .
$$

The pseudopotential approximation is first used to calculate the pseudo-atom energy of lithium, sodium and magnesium. From Table 4, it is clear that we are able to replicate the results obtained by Nogueira et al. (1996). These pseudo-atom energies are further utilized to evaluate the binding energy and bond length of their respective dimers. As is evident from Table 5, the results obtained are in reasonable agreement with previous calculations (Nogueira et al. (1996)).

Table 4

$\underline{\text { Pseudo-atom energy (eV) using the 'Evanescent Core' pseudopotential }}$

\begin{tabular}{ccc}
\hline Metal & KS-DFT-FE (This work) & Nogueira et al. (1996) \\
\hline $\mathrm{Li}$ & -5.97 & -5.97 \\
$\mathrm{Na}$ & -5.21 & -5.21 \\
$\mathrm{Mg}$ & -23.05 & -23.06 \\
\hline
\end{tabular}

Table 5

Binding energy and bond length for selected metal dimers using 'Evanescent Core' pseudopoten$\underline{\text { tial }}$

\begin{tabular}{cccc}
\hline Dimer & Property & KS-DFT-FE (This work) & Nogueira et al. (1996) \\
\hline $\mathrm{Li}_{2}$ & Binding energy (eV) & -0.49 & -0.52 \\
& Bond length (a.u) & 4.86 & 4.92 \\
$\mathrm{Na}_{2}$ & Binding energy (eV) & -0.35 & -0.46 \\
& Bond length (a.u) & 5.72 & 5.77 \\
$\mathrm{Mg}_{2}$ & Binding energy (eV) & -0.06 & -0.04 \\
& Bond length (a.u) & 7.12 & 7.18 \\
\hline
\end{tabular}

Next, we study the properties of some sodium clusters, in particular $1 \times 1 \times 1,2 \times 2 \times 2$ and $3 \times 3 \times 3$ Body Centered Cubic (BCC) unit cells. A representative triangulation $\mathcal{T}_{0}$ used for $2 \times 2 \times 2$ BCC unit cells and its close-up view is shown in Figs. 5 and 6 , respectively. We calculate the binding energy per atom and lattice constant for these clusters by computing the energy for various lattice distances and subsequently fitting the data to a cubic polynomial to obtain the point of minimum. The results so obtained are presented in Table 6. Also, the contours of electron density on the mid-plane and quarterplane of $2 \times 2 \times 2$ BCC unit cells are shown in Figs. 7 and 8, respectively. 


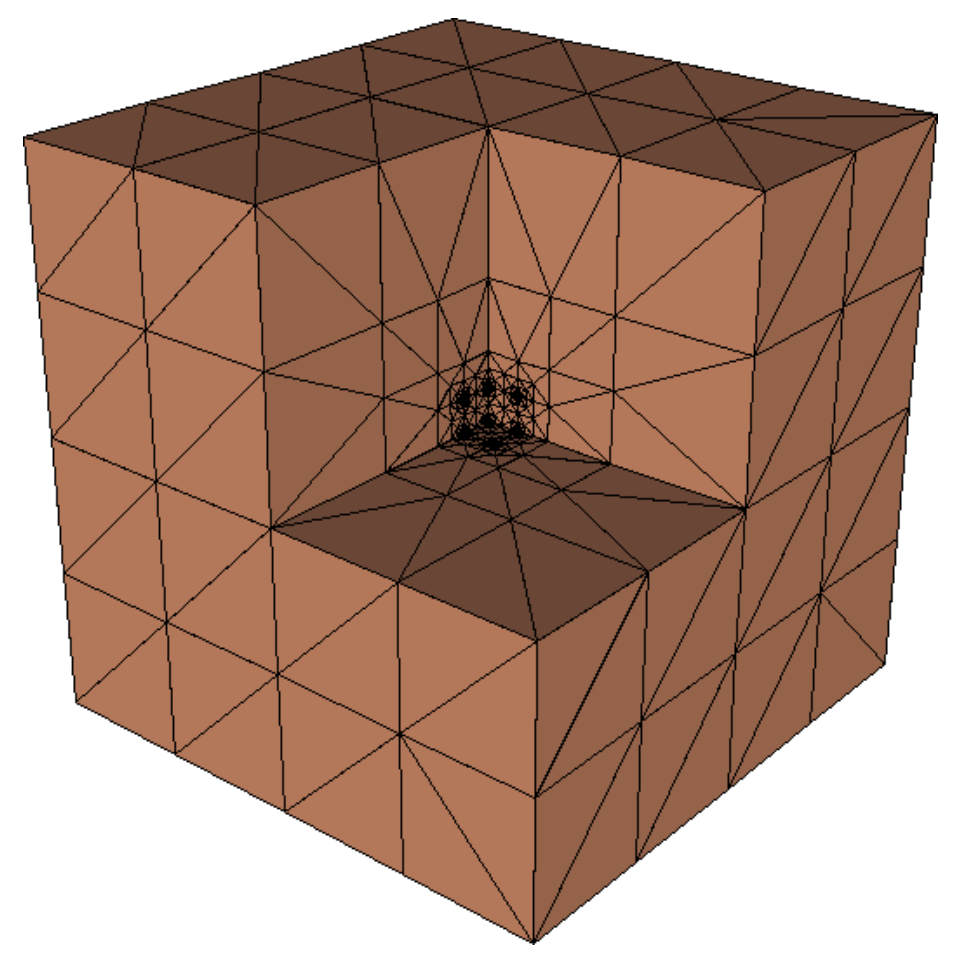

Fig. 5. The triangulation $\mathcal{T}_{0}$ used for $2 \times 2 \times 2 \mathrm{BCC}$ unit cells.

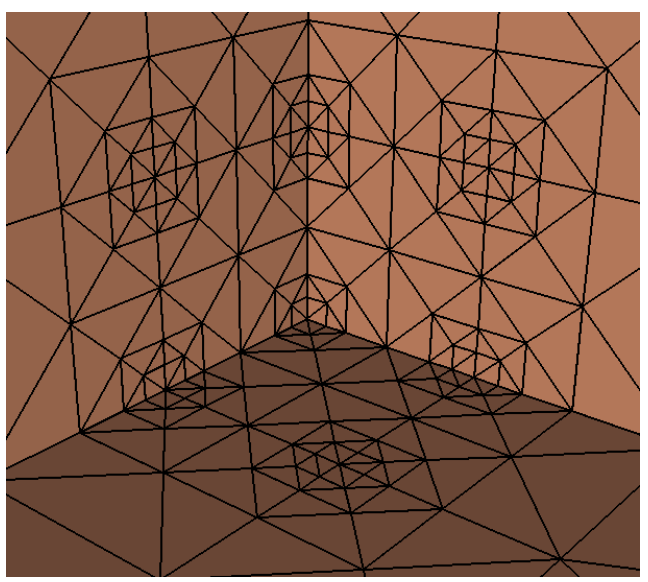

Fig. 6. Close up of the triangulation $\mathcal{T}_{0}$ used for $2 \times 2 \times 2 \mathrm{BCC}$ unit cells.

Table 6

Binding energy per atom and lattice constant of sodium BCC unit cells

\begin{tabular}{cccc}
\hline Property & $1 \times 1 \times 1$ & $2 \times 2 \times 2$ & $3 \times 3 \times 3$ \\
\hline Binding energy/atom (eV) & -0.54 & -0.70 & -0.81 \\
Lattice constant (a.u.) & 7.20 & 7.55 & 7.75 \\
\hline
\end{tabular}




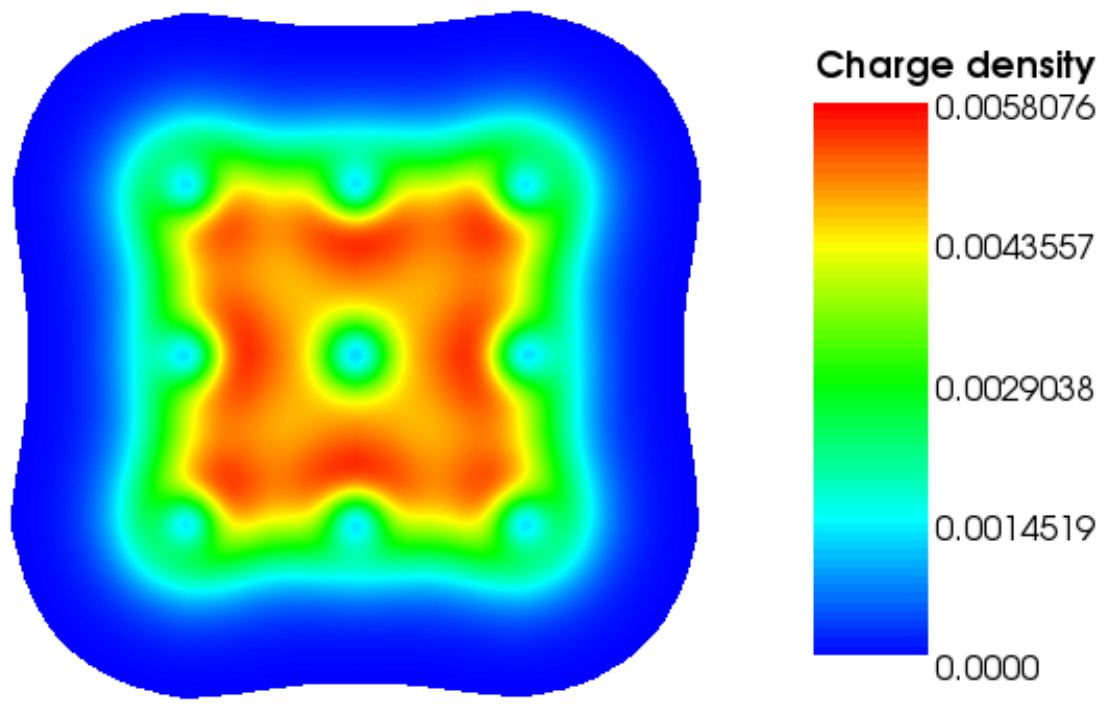

Fig. 7. Contours of electron density on the mid-plane of a sodium cluster with $2 \times 2 \times 2 \mathrm{BCC}$ unit cells.

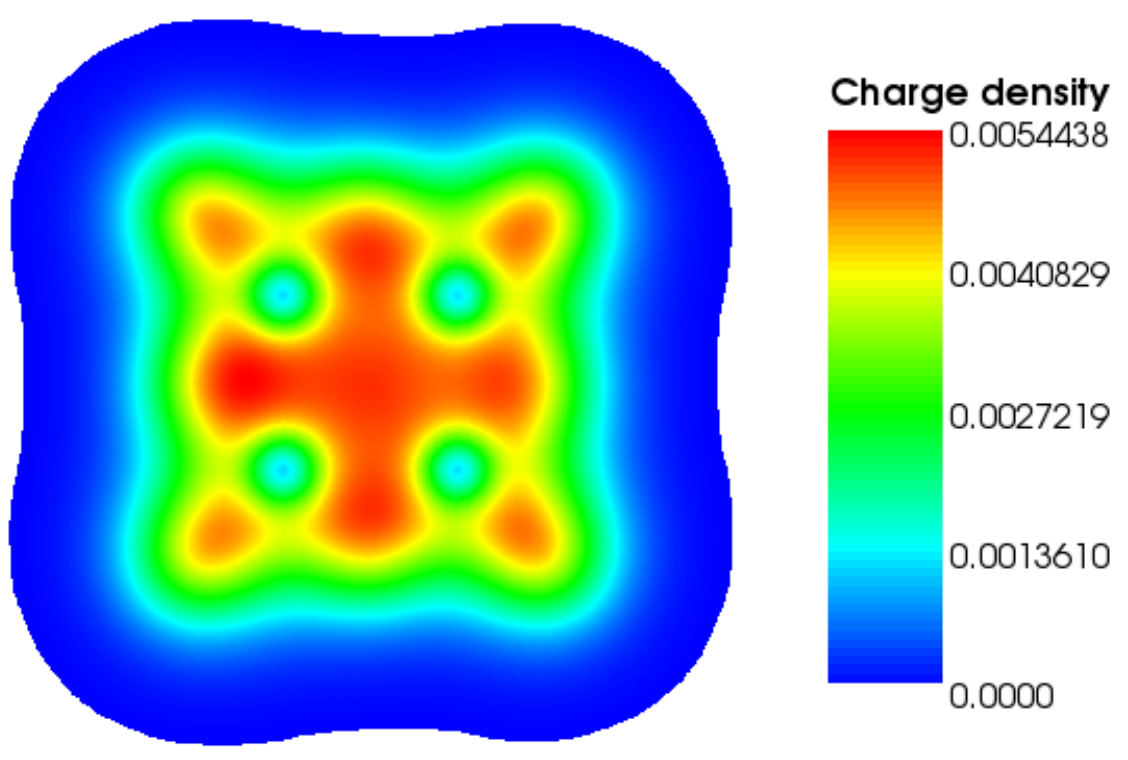

Fig. 8. Contours of electron density on the quarter-plane of a sodium cluster with $2 \times 2 \times 2 \mathrm{BCC}$ unit cells. 


\subsection{Pseudopotential approximation (non-local)}

Norm-conserving pseudopotentials are attractive because of their accuracy, transferability and availability for all elements in the periodic table. Here, we look at the results obtained using the Troullier-Martins (TM) pseudopotential (Troullier and Martins (1991)) implemented in the Kleinman-Bylander form.

The radial component of the TM pseudo-wavefunction for an atom located at $\mathbf{R}_{I}$ is given by

$$
w_{l}(r)=\left\{\begin{array}{l}
w_{l}^{A E}(r), \quad r \geq r_{c}, \\
r^{l} \exp [p(r)], \quad r \leq r_{c},
\end{array}\right.
$$

and the angular momentum dependent pseudopotential component has the form

$$
V_{l}(r)=\left\{\begin{array}{l}
V^{A E}(r), \quad r \geq r_{c}, \\
\epsilon_{l}+\frac{l+1}{r} \frac{p^{\prime}(r)}{2}+\frac{p^{\prime \prime}(r)+\left(p^{\prime}(r)\right)^{2}}{2}, \quad r \leq r_{c} .
\end{array}\right.
$$

The superscript $A E$ stands for 'all-electron' calculation, $\epsilon_{l}$ represents the valence eigenvalues, $r=\left|\mathbf{x}-R_{I}\right|$ and $r_{c}$ is the core radius. The coefficients of the polynomial $p(r)=c_{0}+c_{2} r^{2}+c_{4} r^{4}+c_{6} r^{6}+c_{8} r^{8}+c_{10} r^{10}+c_{12} r^{12}$ are determined by norm conservation, continuity of the pseudo-wavefunction and its first four derivatives and the zero curvature of the pseudopotential at the origin.

In Tables 7, 8 we list the binding energy, bond length of $\mathrm{B}_{2}$ and $\mathrm{C}_{2}$ obtained using the TM pseudopotential and compare them with previous such studies (Engel et al. (2001)). The agreement is good, thereby validating the accuracy of our finite-element implementation.

Table 7

Binding energy and bond length of $\mathrm{B}_{2}$

\begin{tabular}{ccc}
\hline Property & KS-DFT-FE (This work) & Engel et al. (2001) \\
\hline Binding energy (eV) & -3.74 & -3.79 \\
Bond length (a.u.) & 3.00 & 3.02 \\
\hline
\end{tabular}

Table 8

Binding energy and bond length of $\mathrm{C}_{2}$

\begin{tabular}{ccc}
\hline Property & KS-DFT-FE (This work) & Engel et al. (2001) \\
\hline Binding energy (eV) & -6.86 & -6.92 \\
Bond length (a.u.) & 2.35 & 2.35 \\
\hline
\end{tabular}




\subsection{Performance of the numerical method}

This section is devoted to the assessment of the numerical performance of the implementation, specifically regarding the convergence rate with mesh size, scaling of the execution time with problem size and parallel scalability. We begin by assessing the rate of convergence of the finite-element method with decreasing mesh size. From Fig. 9, which depicts the normalized energy-error as a function of mesh size, we obtain $\left|\mathcal{E}^{h}-\mathcal{E}_{0}\right| \propto h^{1.9}$, where $\mathcal{E}_{0}$ is the converged value of the energy obtained by fitting the data to $\mathcal{E}=\mathcal{E}_{0}+C h^{n}$. This analysis thus shows that the energy converges quadratically, which is the expected rate of convergence for linear interpolation (Ciarlet (2002)).

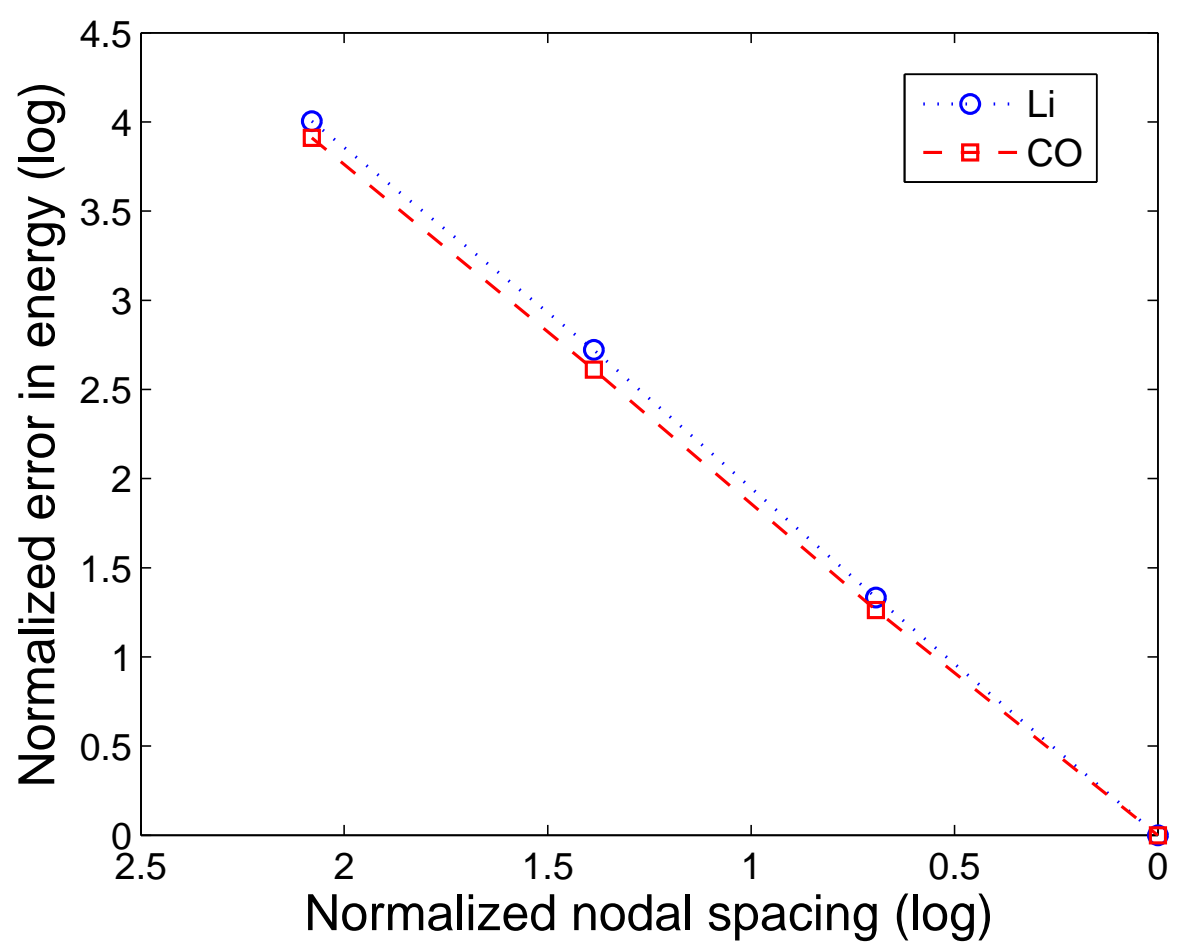

Fig. 9. Convergence rate of the finite-element method.

Fig. 10 collects execution times as a function of the number of nodes in the triangulation for two selected examples. As is evident from the figure, the execution time scales linearly, or $O(N)$, asymptotically with the size $N$ of the problem. This scaling is expected, since the complexity of the solution procedure is dominated by the complexity of the solution of a banded system of equations, which is $O(N)$ when the system bandwidth is independent of $N$. This latter property is typical of finite-elements, for which the system bandwidth is determined by the number of nearest neighbors of a typical node. Thus, our formulation achieves linear, or $O(N)$, scaling with respect to the number of nodes in the triangulation for a fixed number of electrons. 


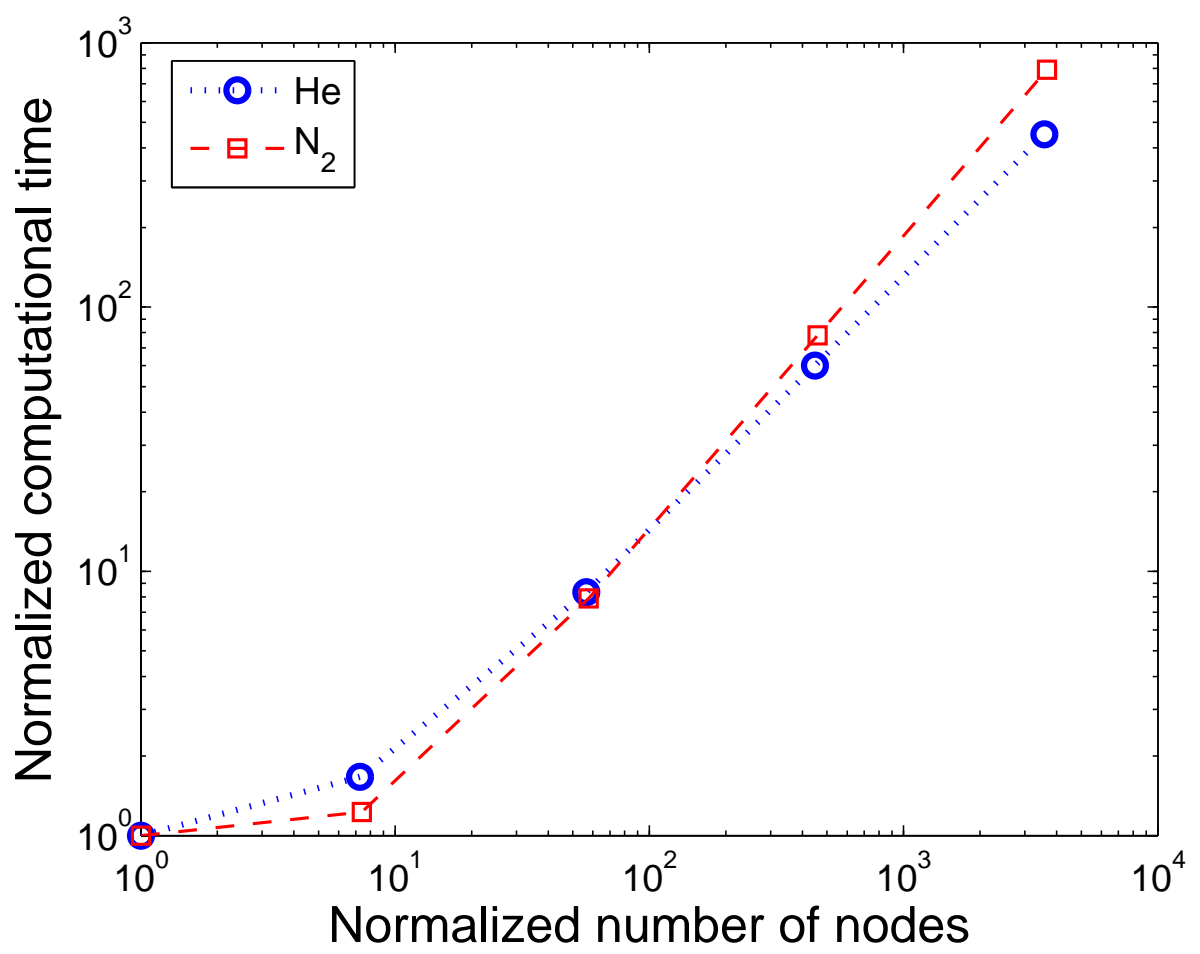

Fig. 10. Scaling of computational time with number of nodes.

Finally, Fig. 11 illustrates the parallel efficiency of the implementation as measured by the relative speedup with increasing number of processors on a fixed problem of constant size. As is evident from the figure, the scaling is roughly linear. However, as the number of processors increases, the parallel efficiency correspondingly decreases owing to the additional communication required between them. Thus, the relative speedup corresponding to a 16-fold increase in the number of processors is of the order of 10.25. This speed-up factor translates into an efficiency of about 0.65, which is in the ballpark expected of domain decomposition applied to medium-size problems. 


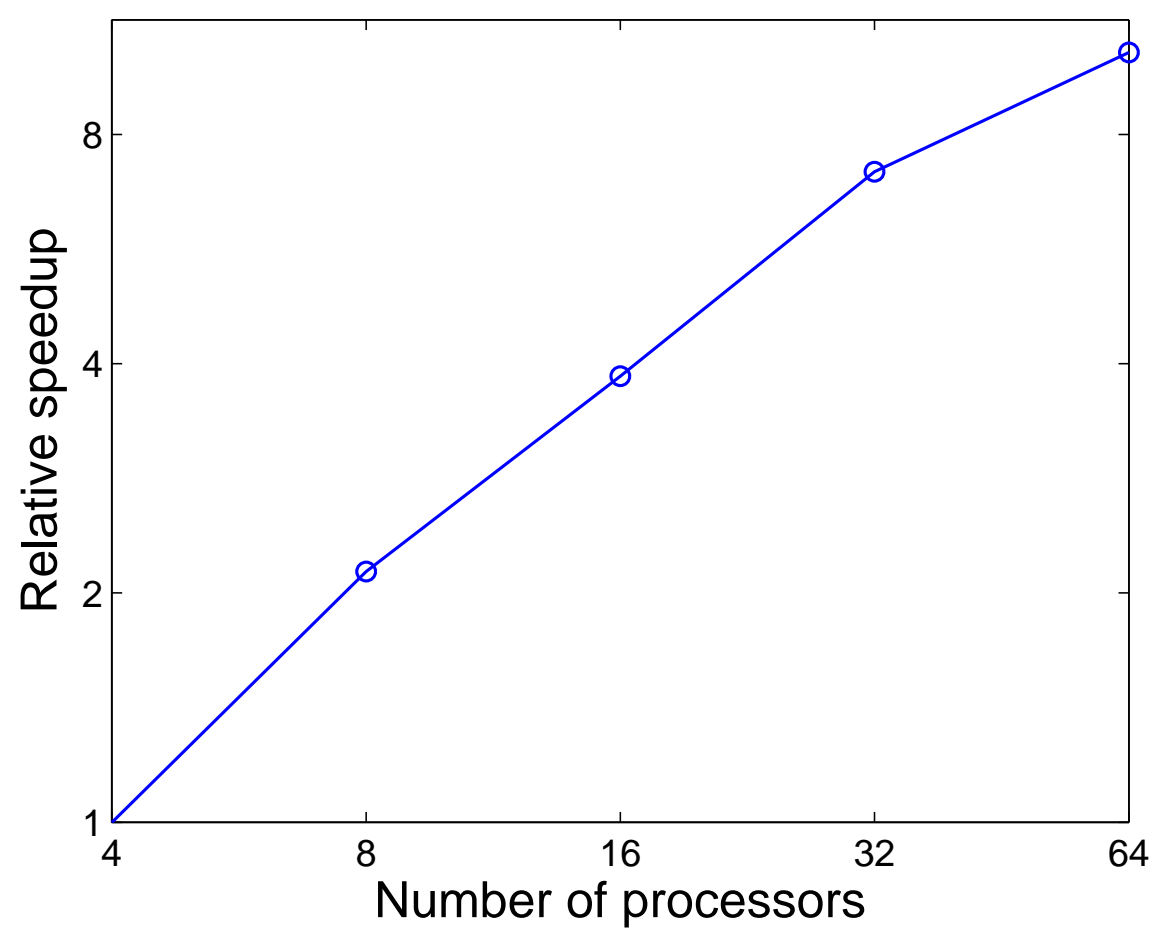

Fig. 11. Relative speedup as a function of the number of processors.

\section{Conclusions}

We have developed a real-space, non-periodic finite-element formulation of KS-DFT. By including the electrostatic potential among the unknown fields we have reformulated the problem as a local saddle-point problem. We have shown the well-posedness of this formulation for both the all-electron problem as well as the pseudopotential approximation. In particular, we have proved the existence of solutions, and, in addition, the convergence of finite-element approximations, including numerical quadratures, using $\Gamma$-convergence methods. We have also developed a parallel implementation of this formulation capable of performing both all-electron and pseudopotential calculations. In this implementation, the advantageous features of both the SCF and Newton's methods have been combined to ensure rapid convergence to the solution. The flexibility provided by the unstructured nature of the finite-element method is exploited by optimally coarsening the triangulation away from the nuclei and by convecting the triangulation with the atomic positions, thereby enhancing the efficiency of the calculations without loss of accuracy. The formulation has been tested through a number of examples and the accuracy of the results is in accord with the literature. Also, various aspects of the numerical performance of the implementation have been investigated, including the convergence rate of the finite-element method and its scaling with increasing system size and number of processors. We obtain ostensibly ideal convergence rate for the finite-element method, linear or $O(N)$-scaling 
with problem size and good parallel scalability.

A shortcoming of the present approach, and of low-order finite-elements in general, is the large number of basis functions required to obtain convergence compared to other bases such as plane waves. This deficiency can be mitigated by using higher-order polynomial interpolation functions (Pask et al. (1999), Pask and Sterne (2005)) or, more generally, other interpolation functions better tailored to the problem. Another drawback of finiteelement based formulations is the difficulty in generating efficient meshes for non-trivial geometries of the atoms. Meshless methods, such as those based on the use of max-ent shape functions (Arroyo and Ortiz (2006)), have the potential to address the preceding concerns simultaneously and thus emerge as worthwhile subjects for future investigation.

As noted in the introduction, this work is intended as a step towards formulating a coarsegrained formulation of KS-DFT capable of analyzing samples of sufficient size to yield meaningful size-independent properties of isolated lattice defects, such as vacancies and dislocations, in concentrations representative of actual material systems. Isolated lattice defects at normal equilibrium concentrations present a unique challenge since the electronic structure of defect cores and the long-range elastic fields of the defects must be characterized simultaneously. As in previous work concerned with OFDFT (Gavini et al. (2007a)), we expect that this goal may effectively be achieved by recourse to the quasicontinuum method as a means of coarse-graining the present real-space KS-DFT formulation. The quasi-continuum coarse-graining of KS-DFT thus naturally suggests itself as another worthwhile subject for future investigation.

\section{Acknowledgements}

We gratefully acknowledge the support of the US Department of Energy through Caltech's ASC/PSAAP Center for the Predictive Modeling and Simulation of High-Energy Density Dynamic Response of Materials. VG also gratefully acknowledges the support of the Air Force Office of Scientific Research under Grant no. FA9550-09-1-0240

\section{References}

Anantharaman, A., Cances, E., 2008. On Kohn-Sham models with LDA and GGA exchange-correlation functionals.

URL http: / /arxiv.org/abs/0809.5139v1

Arroyo, M., Ortiz, M., 2006. Local maximum-entropy approximation schemes: a seamless bridge between finite elements and meshfree methods. International Journal for Numerical Methods in Engineering 65, 2167-2202.

Bachelet, G. B., Hamann, D. R., Schlüter, M., 1982. Pseudopotentials that work: From H to Pu. Physical Review B 26 (8), 4199-4228. 
Bernstein, N., Kermode, J. R., Csanyi, G., 2009. Hybrid atomistic simulation methods for materials systems. Reports on Progress in Physics 72 (2), 026501 (25pp).

Bey, J., Aachen, R., 2000. Simplicial grid refinement: on Freudenthal's algorithm and the optimal number of congruence classes. Numerische Mathematik 85, 1-29.

Bowler, D., Choudhury, R., Gillan, M., Miyazaki, T., 2006. Recent progress with largescale ab initio calculations: the CONQUEST code. Physica Status Solidi B-Basic Solid State Physics 243 (5), 989-1000.

Car, R., Parrinello, M., Nov 1985. Unified approach for molecular dynamics and densityfunctional theory. Phys. Rev. Lett. 55 (22), 2471-2474.

Castro, A., Appel, H., Oliveira, M., Rozzi, C. A., Andrade, X., Lorenzen, F., Marques, M. A. L., Gross, E. K. U., Rubio, A., 2006. octopus: a tool for the application of timedependent density functional theory. Physica Status Solidi B-Basic Solid State Physics 243 (11), 2465-2488.

Ceperley, D. M., Alder, B. J., 1980. Ground state of the electron gas by a stochastic method. Physical Review Letters 45 (7), 566-569.

Chelikowsky, J. R., Troullier, N., Saad, Y., 1994. Finite-difference-pseudopotential method: Electronic structure calculations without a basis. Physical Review Letters $72(8), 1240-1243$.

Choly, N., Lu, G., E, W., Kaxiras, E., Mar 2005. Multiscale simulations in simple metals: A density-functional-based methodology. Phys. Rev. B 71 (9), 094101.

Ciarlet, P., 2002. The finite-element method for elliptic problems. SIAM, Philadelphia.

Dal Maso, G., 1993. An introduction to $\Gamma$-convergence. Birkhäuser, Boston.

Dennis, J., Schnabel, R., 1996. Numerical Methods for Unconstrained Optimization and Nonlinear Equations. SIAM, Philadelphia.

Engel, E., Höck, A., Schmid, R. N., Dreizler, R. M., Chetty, N., 2001. Role of the corevalence interaction for pseudopotential calculations with exact exchange. Physical Review B 64 (12), 125111.

Finnis, M., 2003. Interatomic forces in condensed matter. Oxford University Press.

Fiolhais, C., Perdew, J. P., Armster, S. Q., MacLaren, J. M., Brajczewska, M., 1995. Dominant density parameters and local pseudopotentials for simple metals. Physical Review B 51 (20), 14001-14011.

Freund, R. W., 1993. A transpose-free quasi-minimal residual algorithm for nonhermitian linear systems. SIAM Journal on Scientific Computing 14 (2), 470-482.

Galli, G., Parrinello, M., Dec 1992. Large scale electronic structure calculations. Phys. Rev. Lett. 69 (24), 3547-3550.

García-Cervera, C. J., Lu, J., E, W., Dec 2007. A sub-linear scaling algorithm for computing the electronic structure of materials. Communications in Mathematical Sciences 5 (4), 999-1026.

Garcia-Cervera, C. J., Lu, J., Xuan, Y., E, W., 2009. Linear-scaling subspace-iteration algorithm with optimally localized nonorthogonal wave functions for kohn-sham density functional theory. Physical Review B (Condensed Matter and Materials Physics) 79 (11), 115110.

Gavini, V., Bhattacharya, K., Ortiz, M., 2007a. Quasi-continuum orbital-free densityfunctional theory: A route to multi-million atom non-periodic DFT calculation. Journal of the Mechanics and Physics of Solids 55 (4), $697-718$. 
Gavini, V., Knap, J., Bhattacharya, K., Ortiz, M., 2007b. Non-periodic finite-element formulation of orbital-free density functional theory. Journal of the Mechanics and Physics of Solids 55 (4), $669-696$.

Gilbarg, D., Trudinger, N., 1983. Elliptic differential equations of second order. Springer, New York.

Goedecker, S., Jul 1999. Linear scaling electronic structure methods. Rev. Mod. Phys. 71 (4), 1085-1123.

Gonze, X., Beuken, J. M., Caracas, R., Detraux, F., Fuchs, M., Rignanese, G. M., Sindic, L., Verstraete, M., Zerah, G., Jollet, F., Torrent, M., Roy, A., Mikami, M., Ghosez, P., Raty, J. Y., Allan, D. C., 2002. First-principles computation of material properties: the abinit software project. Computational Materials Science 25, 478-492(15).

Govind, N., Wang, Y. A., Carter, E. A., 1999. Electronic-structure calculations by firstprinciples density-based embedding of explicitly correlated systems. The Journal of Chemical Physics 110 (16), 7677-7688.

Gygi, F. m. c., Galli, G., Jul 1995. Real-space adaptive-coordinate electronic-structure calculations. Physical Review B 52 (4), R2229-R2232.

Hehre, W. J., Stewart, R. F., Pople, J. A., 1969. Self-consistent molecular-orbital methods. I. Use of Gaussian Expansions of Slater-Type Atomic Orbitals. The Journal of Chemical Physics 51 (6), 2657-2664.

Hohenberg, P., Kohn, W., 1964. Inhomogeneous electron gas. Physical Review 136 (3B), B864-B871.

Huber, K., 1972. Constants of diatomic molecules. McGraw-Hill, New York.

Ismail-Beigi, S., Arias, T. A., 2000. New algebraic formulation of density functional calculation. Computer Physics Communications 128 (1-2), 1 - 45.

Kleinman, L., Bylander, D. M., 1982. Efficacious form for model pseudopotentials. Physical Review Letters 48 (20), 1425-1428.

Kohn, W., 1999. Nobel lecture: Electronic structure of matter-wave functions and density functionals. Reviews of Modern Physics 71 (5), 1253-1266.

Kohn, W., Sham, L. J., 1965. Self-consistent equations including exchange and correlation effects. Physical Review 140 (4A), A1133-A1138.

Kotochigova, S., Levine, Z. H., Shirley, E. L., Stiles, M. D., Clark, C. W., 1997. Localdensity-functional calculations of the energy of atoms. Physical Review A 55 (1), 191199.

Kresse, G., Furthmüller, J., 1996. Efficient iterative schemes for ab initio total-energy calculations using a plane-wave basis set. Physical Review B 54 (16), 11169-11186.

Langreth, D. C., Mehl, M. J., 1983. Beyond the local-density approximation in calculations of ground-state electronic properties. Physical Review B 28 (4), 1809-1834.

Lu, G., Tadmor, E. B., Kaxiras, E., 2006. From electrons to finite elements: A concurrent multiscale approach for metals. Physical Review B (Condensed Matter and Materials Physics) 73 (2), 024108.

Mauri, F., Galli, G., Car, R., Apr 1993. Orbital formulation for electronic-structure calculations with linear system-size scaling. Phys. Rev. B 47 (15), 9973-9976.

Modine, N. A., Zumbach, G., Kaxiras, E., Apr 1997. Adaptive-coordinate real-space electronic-structure calculations for atoms, molecules, and solids. Physical Review B 55 (16), 10289-10301. 
Nogueira, F., Fiolhais, C., He, J., Perdew, J. P., Rubio, A., 1996. Transferability of a local pseudopotential based on solid-state electron density. Journal of Physics: Condensed Matter 8 (3), 287-302.

Parr, R., Yang, W., 1989. Density-functional theory of atoms and molecules. Oxford University Press.

Pask, J. E., Klein, B. M., Fong, C. Y., Sterne, P. A., 1999. Real-space local polynomial basis for solid-state electronic-structure calculations: A finite-element approach. Physical Review B 59 (19), 12352-12358.

Pask, J. E., Sterne, P. A., 2005. Finite element methods in ab initio electronic structure calculations. Modelling and Simulation in Materials Science and Engineering 13 (3), R71-R96.

Payne, M. C., Teter, M. P., Allan, D. C., Arias, T. A., Joannopoulos, J. D., 1992. Iterative minimization techniques for ab initio total-energy calculations: molecular dynamics and conjugate gradients. Reviews of Modern Physics 64 (4), 1045-1097.

Perdew, J. P., Chevary, J. A., Vosko, S. H., Jackson, K. A., Pederson, M. R., Singh, D. J., Fiolhais, C., 1992. Atoms, molecules, solids, and surfaces: Applications of the generalized gradient approximation for exchange and correlation. Physical Review B 46 (11), 6671-6687.

Perdew, J. P., Wang, Y., 1992. Accurate and simple analytic representation of the electrongas correlation energy. Physical Review B 45 (23), 13244-13249.

Perdew, J. P., Zunger, A., 1981. Self-interaction correction to density-functional approximations for many-electron systems. Physical Review B 23 (10), 5048-5079.

Pickett, W. E., 1989. Pseudopotential methods in condensed matter applications. Computer Physics reports 9 (3), 115 - 197.

Rappe, A. M., Rabe, K. M., Kaxiras, E., Joannopoulos, J. D., 1990. Optimized pseudopotentials. Physical Review B 41 (2), 1227-1230.

Roothaan, C. C. J., 1951. New developments in molecular orbital theory. Reviews of Modern Physics 23 (2), 69-89.

Rudin, W., 1991. Functional Analysis. Mc Graw Hill.

Saad, Y., 1992. Numerical Methods for Large Eigenvalue Problems. Manchester University Press.

Saad, Y., Schultz, M. H., 1986. GMRES: A Generalized Minimal Residual Algorithm for Solving Nonsymmetric Linear Systems. SIAM Journal on Scientific and Statistical Computing 7 (3), 856-869.

Segall, M. D., Lindan, P. J. D., Probert, M. J., Pickard, C. J., Hasnip, P. J., Clark, S. J., Payne, M. C., 2002. First-principles simulation: ideas, illustrations and the CASTEP code. Journal of Physics: Condensed Matter 14 (11), 2717-2744.

Skylaris, C.-K., Haynes, P. D., Mostofi, A. A., Payne, M. C., 2005. Introducing ONETEP: Linear-scaling density functional simulations on parallel computers. The Journal of Chemical Physics 122 (8), 084119.

Soler, J. M., Artacho, E., Gale, J. D., García, A., Junquera, J., Ordejón, P., SánchezPortal, D., 2002. The siesta method for ab initio order-n materials simulation. Journal of Physics: Condensed Matter 14 (11), 2745-2779.

Struwe, M., 1990. Variational Methods, Applications to Nonlinear Partial Differential Equations and Hamiltonian Systems. Springer, Berlin. 
Thoutireddy, P., 2002. Variational arbitrary lagrangian-eulerian method. Ph.D. thesis, California Institute of Technology.

Troullier, N., Martins, J. L., 1991. Efficient pseudopotentials for plane-wave calculations. Physical Review B 43 (3), 1993-2006.

Tsuchida, E., 2004. Ab initio molecular-dynamics study of liquid formamide. The Journal of Chemical Physics 121 (10), 4740-4746.

van der Vorst, H. A., 1992. BI-CGSTAB: a fast and smoothly converging variant of BICG for the solution of nonsymmetric linear systems. SIAM Journal on Scientific and Statistical Computing 13 (2), 631-644.

Vanderbilt, D., 1990. Soft self-consistent pseudopotentials in a generalized eigenvalue formalism. Physical Review B 41 (11), 7892-7895.

Veillard, A., Clementi, E., 1968. Correlation energy in atomic systems. v. degeneracy effects for the second-row atoms. The Journal of Chemical Physics 49 (5), 2415-2421.

Wills, J. M., Cooper, B. R., 1987. Synthesis of band and model hamiltonian theory for hybridizing cerium systems. Physical Review B 36 (7), 3809-3823.

Zumbach, G., Modine, N. A., Kaxiras, E., 1996. Adaptive coordinate, real-space electronic structure calculations on parallel computers. Solid State Communications 99 (2), $57-61$.

\section{Appendix}

\section{A Computation of forces}

For completeness, we derive the forces that determine the equilibrium nuclear configuration. To do so, we assume that for any configuration of the nuclei, the variational problem defined by

$$
\inf _{\Psi \in\left(H_{0}^{1}(\Omega)\right)^{N}} \sup _{\phi \in H_{0}^{1}(\Omega)} \mathcal{E}(\Psi, \phi, \mathbf{R})
$$

subject to the constraints given by Eqn. (21), has been solved. The derivation below closely follows Thoutireddy (2002); Gavini et al. (2007a). Using Lagrange multipliers to enforce the constraints, we have

$$
\begin{array}{r}
\mathcal{E}_{c}\left(\Psi, \phi, \mathbf{R}, \Lambda^{\alpha}, \Lambda^{\beta}\right)=\int_{\Omega} f(\Psi, \nabla \Psi) \mathrm{d} \mathbf{x}+\int_{\Omega}(\rho(\mathbf{x})+b(\mathbf{x}, \mathbf{R})) \phi(\mathbf{x}, \mathbf{R}) \mathrm{d} \mathbf{x} \\
-\frac{1}{8 \pi} \int_{\Omega}|\nabla \phi(\mathbf{x}, \mathbf{R})|^{2} \mathrm{~d} \mathbf{x}-\sum_{i=1}^{N_{\alpha}} \sum_{j=1}^{N_{\alpha}} \lambda_{i j}^{\alpha}\left(\int_{\Omega} \psi_{i \alpha}^{*}(\mathbf{x}) \psi_{j \alpha}(\mathbf{x}) \mathrm{d} \mathbf{x}-\delta_{i j}\right) \\
-\sum_{i=1}^{N_{\beta}} \sum_{j=1}^{N_{\beta}} \lambda_{i j}^{\beta}\left(\int_{\Omega} \psi_{i \beta}^{*}(\mathbf{x}) \psi_{j \beta}(\mathbf{x}) \mathrm{d} \mathbf{x}-\delta_{i j}\right) .
\end{array}
$$

In this situation, consider $I_{1}^{h}+I_{2}^{h}+I_{3}^{h}+I_{4}^{h}$, where 


$$
\begin{aligned}
I_{1}^{h} & =\int_{\Omega} f(\Psi, \nabla \Psi) \mathrm{d} \mathbf{x}, \\
I_{2}^{h} & =\int_{\Omega}(\rho(\mathbf{x})+b(\mathbf{x}, \mathbf{R})) \phi(\mathbf{x}, \mathbf{R}) \mathrm{d} \mathbf{x}, \\
I_{3}^{h} & =-\frac{1}{8 \pi} \int_{\Omega}|\nabla \phi(\mathbf{x}, \mathbf{R})|^{2} \mathrm{~d} \mathbf{x}, \\
I_{4}^{h} & =-\sum_{i=1}^{N_{\alpha}} \sum_{j=1}^{N_{\alpha}} \lambda_{i j}^{\alpha}\left(\int_{\Omega} \psi_{i \alpha}^{*}(\mathbf{x}) \psi_{j \alpha}(\mathbf{x}) \mathrm{d} \mathbf{x}-\delta_{i j}\right)-\sum_{i=1}^{N_{\beta}} \sum_{j=1}^{N_{\beta}} \lambda_{i j}^{\beta}\left(\int_{\Omega} \psi_{i \beta}^{*}(\mathbf{x}) \psi_{j \beta}(\mathbf{x}) \mathrm{d} \mathbf{x}-\delta_{i j}\right) .
\end{aligned}
$$

Note that

$$
I_{1}^{h}=\int_{\Omega} f(\Psi, \nabla \Psi) \mathrm{d} \mathbf{x}=\sum_{e \in T_{h}} \int_{\hat{\Omega}} f(\Psi, \nabla \Psi) \operatorname{det}\left(\frac{\partial \mathbf{x}_{M}}{\partial \hat{\mathbf{x}}_{N}}\right) \mathrm{d} \hat{\mathbf{x}}
$$

where $\hat{\Omega}$ is the reference volume in isoparametric formulation and $\frac{\partial \mathbf{x}_{M}}{\partial \hat{\mathbf{x}}_{N}}$ is the Jacobian of the transformation. Taking first variations of $I_{1}$, we obtain

$$
\begin{aligned}
\delta I_{1}^{h}=\sum_{e \in \mathcal{T}_{0}} \int_{\hat{\Omega}}\left\{-\sum_{\sigma} \sum_{i=1}^{N_{\sigma}} \frac{\delta f(\Psi, \nabla \Psi)}{\delta \psi_{i \sigma, J}}\left[\sum_{a=1}^{N_{h}} \psi_{i \sigma}^{a} \hat{N}_{a, A} \frac{\partial \hat{\mathbf{x}}_{A}}{\partial \mathbf{x}_{K}}\left(\sum_{b=1}^{N_{h}} \delta \mathbf{x}_{b K}^{e} \hat{N}_{b, B}\right) \frac{\partial \hat{\mathbf{x}}_{B}}{\partial \mathbf{x}_{J}}\right]\right. \\
\left.+f(\Psi, \nabla \Psi)\left(\sum_{b=1}^{N_{h}} \delta \mathbf{x}_{b K}^{e} \hat{N}_{b, B}\right) \frac{\partial \hat{\mathbf{x}}_{B}}{\partial \mathbf{x}_{K}}\right\} \operatorname{det}\left(\frac{\partial \mathbf{x}_{M}}{\partial \hat{\mathbf{x}}_{N}}\right) \mathrm{d} \hat{\mathbf{x}} \\
=\sum_{e \in \mathcal{T}_{0}} \int_{\Omega^{e}}\left\{-\sum_{\sigma} \sum_{i=1}^{N_{\sigma}} \frac{\delta f(\Psi, \nabla \Psi)}{\delta \psi_{i \sigma, J}}\left[\sum_{a=1}^{N_{h}} \psi_{i \sigma}^{a} N_{a, K}\right]+f(\Psi, \nabla \Psi) \delta_{K J}\right\}\left(\sum_{b=1}^{N_{h}} \delta \mathbf{x}_{b K}^{e} N_{b, J}\right) \mathrm{d} \mathbf{x} \\
=\sum_{e \in \mathcal{T}_{0}} \int_{\Omega^{e}}\left\{-\sum_{\sigma} \sum_{i=1}^{N_{\sigma}} \frac{\delta f(\Psi, \nabla \Psi)}{\delta \psi_{i \sigma, J}} \psi_{i \sigma, K}(\mathbf{x})+f(\Psi, \nabla \Psi) \delta_{K J}\right\}\left(\sum_{b=1}^{N_{h}} \delta \mathbf{x}_{b K}^{e} N_{b, J}\right) \mathrm{d} \mathbf{x} .
\end{aligned}
$$

Similarly, note that

$$
I_{2}^{h}=\int_{\Omega}(\rho(\mathbf{x})+b(\mathbf{x}, \mathbf{R})) \phi(\mathbf{x}, \mathbf{R}) \mathrm{d} \mathbf{x}=\sum_{e \in \mathcal{T}_{0}} \int_{\hat{\Omega}}(\rho(\mathbf{x})+b(\mathbf{x}, \mathbf{R})) \phi(\mathbf{x}, \mathbf{R}) \operatorname{det}\left(\frac{\partial \mathbf{x}_{M}}{\partial \hat{\mathbf{x}}_{N}}\right) \mathrm{d} \hat{\mathbf{x}}
$$

Taking variations, we find 


$$
\begin{aligned}
\delta I_{2}^{h}= & \sum_{e \in \mathcal{T}_{0}} \int_{\hat{\Omega}}(\rho(\mathbf{x})+b(\mathbf{x}, \mathbf{R})) \phi(\mathbf{x}, \mathbf{R})\left(\sum_{b=1}^{N_{h}} \delta \mathbf{x}_{b K}^{e} \hat{N}_{b, B}\right) \frac{\partial \hat{\mathbf{x}}_{B}}{\partial \mathbf{x}_{K}} \operatorname{det}\left(\frac{\partial \mathbf{x}_{M}}{\partial \hat{\mathbf{x}}_{N}}\right) \mathrm{d} \hat{\mathbf{x}} \\
& +\sum_{e \in \mathcal{T}_{0}} \int_{\hat{\Omega}}(\delta b) \phi(\mathbf{x}, \mathbf{R}) \operatorname{det}\left(\frac{\partial \mathbf{x}_{M}}{\partial \hat{\mathbf{x}}_{N}}\right) \mathrm{d} \hat{\mathbf{x}} \\
= & \sum_{e \in \mathcal{T}_{0}} \int_{\Omega^{e}}(\rho(\mathbf{x})+b(\mathbf{x}, \mathbf{R})) \phi(\mathbf{x}, \mathbf{R}) \delta_{K J}\left(\sum_{b=1}^{N_{h}} \delta \mathbf{x}_{b K}^{e} N_{b, J}\right) \mathrm{d} \mathbf{x} \\
& +\sum_{e \in \mathcal{T}_{0}} \int_{\Omega^{e}} b(\mathbf{x}, \mathbf{R})\left(\sum_{a=1}^{N_{h}} \phi_{a} N_{a, K}\right) \delta \mathbf{x}_{b K}^{e} \mathrm{~d} \mathbf{x} .
\end{aligned}
$$

Similarly,

$$
\begin{aligned}
\delta I_{3}^{h}= & -\frac{1}{8 \pi} \sum_{e \in \mathcal{T}_{0}} \int_{\Omega^{e}}\left\{|\nabla \phi(\mathbf{x}, \mathbf{R})|^{2} \delta_{K J}-2 \phi_{, J}(\mathbf{x}, \mathbf{R}) \phi_{, K}(\mathbf{x}, \mathbf{R})\right\}\left(\sum_{b=1}^{N_{h}} \delta \mathbf{x}_{b K}^{e} N_{b, J}\right) \mathrm{d} \mathbf{x}, \\
\delta I_{4}^{h}= & -\sum_{i=1}^{N_{\alpha}} \sum_{j=1}^{N_{\alpha}} \lambda_{i j}^{\alpha} \sum_{e \in \mathcal{T}_{0}} \int_{\Omega^{e}} \psi_{i \alpha}^{*}(\mathbf{x}) \psi_{j \alpha}(\mathbf{x}) \delta_{K J}\left(\sum_{b=1}^{N_{h}} \delta \mathbf{x}_{b K}^{e} N_{b, J}\right) \mathrm{d} \mathbf{x} \\
& -\sum_{i=1}^{N_{\beta}} \sum_{j=1}^{N_{\beta}} \lambda_{i j}^{\beta} \sum_{e \in \mathcal{T}_{0}} \int_{\Omega^{e}} \psi_{i \beta}^{*}(\mathbf{x}) \psi_{j \beta}(\mathbf{x}) \delta_{K J}\left(\sum_{b=1}^{N_{h}} \delta \mathbf{x}_{b K}^{e} N_{b, J}\right) \mathrm{d} \mathbf{x} .
\end{aligned}
$$

Collecting all terms, the force on the $\mathrm{b}^{\text {th }}$ node along the $K^{\text {th }}$ direction is given by

$$
f^{b K}=\sum_{e \in \mathcal{T}_{0}} \int_{\Omega^{e}} Z_{b} b_{b}\left(\sum_{a=1}^{N_{h}} \phi_{a} N_{a, K}\right) \mathrm{d} \mathbf{x}+\sum_{e \in \mathcal{T}_{0}} \int_{\Omega^{e}} \mathrm{E}_{K J} N_{b, J} \mathrm{~d} \mathbf{x},
$$

where

$$
\begin{aligned}
& \mathrm{E}_{K J}=\left\{f(\Psi, \nabla \Psi)+(\rho(\mathbf{x})+b(\mathbf{x}, \mathbf{R})) \phi(\mathbf{x}, \mathbf{R})-\frac{1}{8 \pi}|\nabla \phi(\mathbf{x}, \mathbf{R})|^{2}\right\} \delta_{K J} \\
& -\left\{\sum_{i=1}^{N_{\alpha}} \sum_{j=1}^{N_{\alpha}} \lambda_{i j}^{\alpha} \psi_{i \alpha}^{*}(\mathbf{x}) \psi_{j \alpha}(\mathbf{x})+\sum_{i=1}^{N_{\beta}} \sum_{j=1}^{N_{\beta}} \lambda_{i j}^{\beta} \psi_{i \beta}^{*}(\mathbf{x}) \psi_{j \beta}(\mathbf{x})\right\} \delta_{K J} \\
& -\sum_{\sigma} \sum_{i=1}^{N_{\sigma}} \frac{\delta f(\Psi, \nabla \Psi)}{\delta \psi_{i \sigma, J}} \psi_{i \sigma, K}(\mathbf{x})+\frac{1}{4 \pi} \phi_{, J}(\mathbf{x}, \mathbf{R}) \phi_{, K}(\mathbf{x}, \mathbf{R})
\end{aligned}
$$

Note that the first term of Eqn. (A.3) corresponds to the Hellmann-Feynman force on the nuclei, whereas the second term arises because of the finite-element discretization and acts on all the nodes of the triangulation. The latter arises from the need to minimize the energy with respect to the nodal configuration for a fixed number of nodes. Hence it can be used effectively for a posteriori mesh adaption. Note that the discretization force is local. 